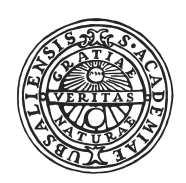

UPPSALA

UNIVERSITET

\title{
Solar Radiation Theory
}

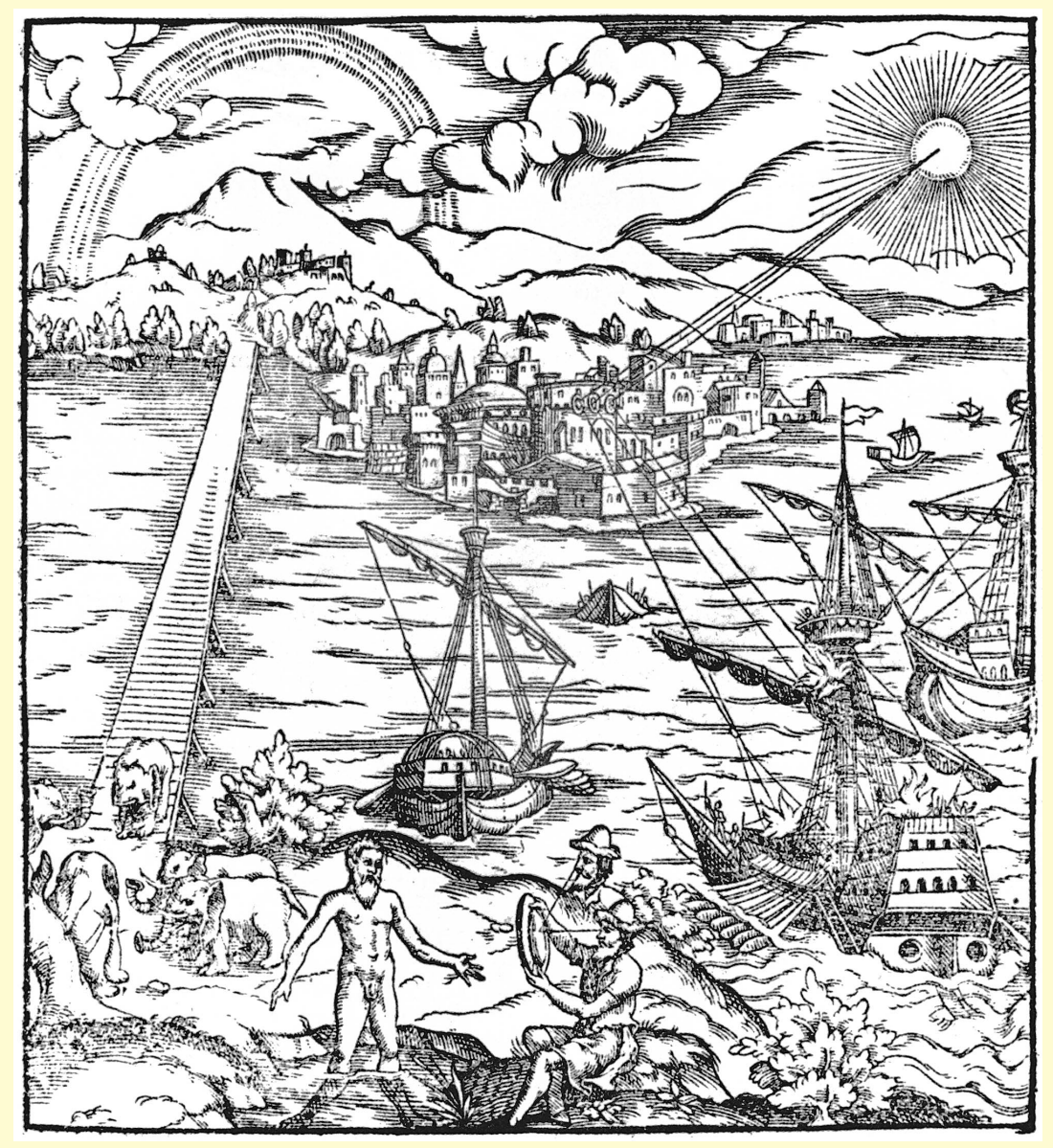

Joakim Widén and Joakim Munkhammar

Department of Engineering Sciences

Uppsala University 
Solar Radiation Theory, Uppsala University 2019.

Copyright (c) Joakim Widén and Joakim Munkhammar 2019.

ISBN 978-91-506-2760-2

DOI $10.33063 /$ diva-381852

URN http://urn.kb.se/resolve?urn=urn:nbn:se:uu:diva-381852

Cover figure: The front page of Opticae Thesaurus from 1572, showing ships set on fire with the help of parabolic mirrors, an application of solar irradiance first attributed by legend to the greek astronomer and mathematician Archimedes. The image also shows other optical phenomena such as a rainbow and distorted images due to refraction in water. (Image from Wikimedia Commons, public domain.) 


\section{Contents}

Preface

$\begin{array}{lll}1 & \text { Introduction } & 7\end{array}$

2 Black-body radiation and the Sun 9

2.1 The source of solar energy . . . . . . . . . . . . . . . 9

2.2 Planck's radiation law . . . . . . . . . . . . . 10

2.3 The solar constant . . . . . . . . . . . . . . . . 13

2.4 Categorization of radiation . . . . . . . . . . . 15

3 Available solar radiation on Earth 17

3.1 Atmospheric attenuation . . . . . . . . . . . 17

3.2 Air mass . . . . . . . . . . . . . . . . . . . . . 18

3.3 Radiation components . . . . . . . . . . . . . . 19

3.4 Temporal and geographical variations in solar energy availability . . . . . . . . . . . . . . . 21

3.5 Earth's energy balance . . . . . . . . . . . . 25

4 Quantifying available solar energy on planar surfaces $\quad 27$

4.1 Measured solar irradiance data . . . . . . . . . . 28

4.2 Solar time . . . . . . . . . . . . . . 30 
4.3 Solar angles . . . . . . . . . . . . . . . . . 31

4.4 Extraterrestrial radiation . . . . . . . . . . . . 33

4.5 Beam radiation on tilted surfaces . . . . . . . . . . 34

4.6 Diffuse radiation on tilted surfaces . . . . . . . . . 35

4.7 Ground-reflected radiation . . . . . . . . . . . . 37

4.8 Complete model for tilted plane global radiation . . . . . 37

4.9 Some notes on optimization of surface orientation . . . 37

$\begin{array}{lll}5 & \text { Exercises } & 39\end{array}$

A Derivation of the incidence angles of beam radiation $\quad 45$

A.1 Zenith angle of incidence . . . . . . . . . . . . . 45

A.2 Incidence angle on an arbitrarily oriented plane . . . . 47 


\section{Nomenclature}

\begin{tabular}{|c|c|c|c|}
\hline Symbol & Description & Value & Unit \\
\hline$a$ & First Ångström coefficient & - & - \\
\hline$A_{i}$ & Anisotropy index & - & - \\
\hline$b$ & Second Ångström coefficient & - & - \\
\hline$c$ & Speed of light in vacuum & $2.998 \times 10^{8}$ & $\mathrm{~ms}^{-1}$ \\
\hline$d$ & Day of year & - & - \\
\hline$f_{\text {view }}$ & View factor & - & - \\
\hline$G_{0 n}, G_{0}$ & $\begin{array}{l}\text { Extraterrestrial radiation, nor- } \\
\text { mal and on horizontal plane }\end{array}$ & - & $\mathrm{Wm}^{-2}$ \\
\hline$G_{b}$ & Total blackbody radiation & - & $\mathrm{Wm}^{-2}$ \\
\hline$G_{b \lambda}$ & $\begin{array}{l}\text { Wavelength distribution of } \\
\text { blackbody radiation }\end{array}$ & - & $\mathrm{Wm}^{-2} \mu \mathrm{m}^{-1}$ \\
\hline$G_{c}$ & Clear-sky irradiance & - & $\mathrm{Wm}^{-2}$ \\
\hline$G_{s c}$ & Solar constant & 1367 & $\mathrm{Wm}^{-2}$ \\
\hline$h$ & Planck's constant & $6.626 \times 10^{-34}$ & Js \\
\hline$H$ & Total monthly irradiation & - & $\mathrm{kWhm}^{-2}$ \\
\hline$H_{c}$ & $\begin{array}{l}\text { Total monthly clear-sky irradia- } \\
\text { tion }\end{array}$ & - & $\mathrm{kWhm}^{-2}$ \\
\hline$I_{0}$ & $\begin{array}{l}\text { Extraterrestrial radiation on } \\
\text { horizontal plane over a time in- } \\
\text { terval }\end{array}$ & - & $\mathrm{Wm}^{-2}$ \\
\hline$I, I_{T}$ & $\begin{array}{l}\text { Global radiation on horizontal } \\
\text { and tilted planes over a time } \\
\text { interval }\end{array}$ & - & $\mathrm{Wm}^{-2}$ \\
\hline
\end{tabular}


Symbol Description

$I_{b}, I_{b T} \quad$ Beam radiation on horizontal and tilted planes over a time interval

$I_{d}, I_{d T}$

Diffuse radiation on horizontal and tilted planes over a time interval

$I_{g T} \quad$ Ground-reflected radiation on tilted plane over a time interval

$k \quad$ Boltzmann's constant

$L_{l o c} \quad$ Local meridian

$L_{s t}$

$m$

$n$

$R_{b}$

$S$

$t_{s}$

$t_{s t}$

$T$

$\beta$

$\delta$

$\gamma$

$\kappa$

$\lambda$

$\omega$

$\phi$

$\Phi_{b \lambda}$

$\rho_{g}$

$\sigma$

$\theta_{z}$

$\theta_{z}$

Standard meridian

Air mass

Refractive index

Geometric factor

Fraction of bright sunshine hours

Solar time

Standard clock time

Temperature

Tilt angle

Declination

Azimuth angle

Wavelength of electromagnetic -

radiation

Hour angle

Latitude

Wavelength distribution of pho- ton flux

Albedo of the ground

Stefan-Boltzmann constant

Angle of incidence on tilted plane

Zenith angle of incidence
Value

Unit

$\mathrm{Wm}^{-2}$

$\mathrm{Wm}^{-2}$

$\mathrm{Wm}^{-2}$

$1.380 \times 10^{-23} \mathrm{JK}^{-1}$

○

$\circ$

$\min$

$\min$

$\mathrm{K}$

Clear-sky index

$\mu \mathrm{m}$

$\mathrm{s}^{-1} \mathrm{~m}^{-2} \mu \mathrm{m}^{-1}$

$5.670 \times 10^{-8}$

$\mathrm{Wm}^{-2} \mathrm{~K}^{-4}$ 


\section{Preface}

One of the challenges in solar engineering is that the availability of the solar resource varies with time and location. An important engineering task is to design solar energy systems that are able to collect as much solar radiation as possible under these constraints. This book introduces the basic properties of solar radiation that are required to understand how the solar resource can be converted into useful heat and electricity, and what the limitations are. It also shows how solar radiation on planar surfaces can be modeled mathematically. This is useful when optimizing the orientation of collecting surfaces and predicting the performance of different system designs. The book builds upon lecture notes from solar engineering courses at Uppsala University, carefully edited to suit a wider scientific and engineering audience. The two authors have, together, more than two decades' experience of teaching, research and development in the field of solar irradiance modeling.

Chapter 1 gives a short historical background to utilization of solar irradiance. Chapter 2 reviews the most important concepts of black-body radiation and electromagnetic radiation. Chapter 3 gives an overview of the availability of the solar resource on Earth. Chapter 4 provides the mathematical framework for modeling solar energy availability on arbitrarily oriented, planar surfaces, based on measurements in the horizontal plane. Review questions on the content as well as exercises are included in chapter 5. Additionally, a derivation of the incidence angles of beam radiation (not commonly explained in the available literature) is presented in the appendix.

Uppsala, April 2019 Joakim Widén and Joakim Munkhammar 


\section{Chapter 1}

\section{Introduction}

The Sun has played an important role in human cultures throughout history. Archaeological sources provide glimpses of sun-worshipping in prehistoric societies and of early astronomical records in Mesopotamian civilizations. The abundance of the Sun's energy and its seasonal availability have naturally set the limits of human life and societal prosperity and growth, governing the turn of seasons and the wheel of the year in agrarian societies. However, direct use of solar radiation for specific purposes such as heating and providing power, made its appearance relatively late in history. The Greeks were aware of various optical phenomena, and Archimedes is said to have used parabolic mirrors, perhaps highly polished bronze or copper shields, to put fire to Roman enemy ships outside Syracuse in 212 B.C., ${ }^{1}$ but more practical use of solar energy came with the scientific revolution [1, p. 386].

Starting in the 17th century, attempts were made not only to concentrate sunlight but to also make it perform mechanical work. In the beginning of the century, french physicist Salomon de Caus constructed the first prototype for a sun-fuelled steam engine using concentrating lenses. However, these devices would be more accurately described as toys than usable machines. In 1861 Augustin Mouchot, a French mathematics teacher,

\footnotetext{
${ }^{1}$ In the 18th century French naturalist Georges Buffon attempted a reconstruction of the Archimedean myth and apparently managed to set fire to an old house using around 150 mirrors at a distance of 60 meters. More modern reconstructions were made both in 1992 and 2005, concluding that the effectiveness of the mirror tactic would have been minor both in terms of manpower and inflicted damage [1].
} 
Chapter 1. Introduction

managed to produce enough steam to drive a small engine. After years of research and development he succeeded in producing an engine large enough to power a printing press, which was presented to the world at the Universal Exhibition in Paris in 1878. Even if widespread use of solar power was to take another one hundred-odd years to emerge, the technology for producing mechanical work with the help of the Sun was in place [1, p. 389].

The technology for producing solar electricity was also in place, by adding a generator to Mouchot's engine. In fact, the first solar power plant went into construction in Egypt in 1912, but after the First World War oil and coal provided more competitive means of producing electric power. Instead, a completely different technology was to harness the solar irradiance on a large scale. Soon after a breakthrough with silicon solar cells at Bell Labs in the U.S. the first commercial photovoltaic (PV) panels, called "Solar Batteries", were produced in the 1950s [1, pp. 392394]. The devices soon found their use in various products, ranging from pocket calculators to satellites, but it was after massive subsidy schemes were introduced in Germany and Japan in the 1990s that rooftop PV systems connected to the power grid began to get widely used. Markets expanded, prices gradually dropped and solar power began making its way into power systems.

For all of these solar-powered devices, the properties of solar radiation set the operational constraints. In the following chapters, we will give you an understanding of basic solar radiation theory and how to use it for quantifying the solar energy on any device, be it a polished bronze mirror or a utility-scale solar power plant. 


\section{Chapter 2}

\section{Black-body radiation and the Sun}

This section reviews the fundamentals of electromagnetic and black-body radiation that are required to understand the nature of the Sun and of the solar radiation that reaches Earth. It also defines some important concepts that are useful for practical applications of solar radiation.

\subsection{The source of solar energy}

The Sun, our closest star, is a spherical gaseous self-gravitating body consisting mainly of hydrogen. It is located at the center of the solar system, on average $1.5 \times 10^{11} \mathrm{~m}$ from Earth. At the inner core of the Sun, the gravitational force creates a pressure which generates nuclear fusion that turns hydrogen into helium. In this process a portion of the mass is converted into an abundant amount of electromagnetic radiation, which makes the Sun the dominant source of radiative energy in the solar system. The Sun has a complex physical structure and consists of several regions, from the dense inner core to the outer atmospherical layer, the corona. Both the corona and the core are very hot, in the order of $10^{6}-10^{7} \mathrm{~K}$, while the intermediate regions that transport and emit energy as outgoing radiation are cooler (although hot by earthly standards).

Energy from fusion reactions in the Sun's interior is transported through successive convection, radiation, absorption, emission and reradiation to the Sun's equivalent of a surface, the photosphere, which absorbs and 
Chapter 2. Black-body radiation and the Sun

emits a continuous spectrum of radiation. The photosphere is the source of most visible radiation reaching Earth. It has a surface temperature, or, more correctly, an effective black-body temperature of $5777 \mathrm{~K}$ (this is the temperature of a black-body radiating the same amount of energy as the Sun). Although it consists of several absorbing and emitting layers and has a considerable temperature gradient across its radius, the Sun closely resembles the ideal concept of a black-body, the properties of which will be explored in the next section.

\subsection{Planck's radiation law}

In order to understand the properties of the solar radiation reaching Earth, it is useful to review some concepts of electromagnetic radiation and the properties of black-bodies. Electromagnetic radiation can be regarded as a wave, characterized by its wavelength $\lambda$. All electromagnetic radiation travels at the speed of light $c\left(2.998 \times 10^{8} \mathrm{~m} / \mathrm{s}\right)$ in a vacuum (or $c / n$ in a material with refractive index $n$ ) and has a frequency $\nu$ such that $c=\lambda \nu$. By quantum mechanics, electromagnetic radiation can also be regarded as a flux of photons, where the energy content in each photon depends on the frequency. In electromagnetic radiation with a certain wavelength $\lambda$ a photon has the energy

$$
E=h \nu=\frac{h c}{\lambda}
$$

where $h$ is Planck's constant $\left(6.626 \times 10^{-34} \mathrm{Js}\right)$.

The radiation emitted from a hot object like the Sun, or any object for that matter, is distributed over a range of wavelengths and, consequently, consists of a flux of photons with different energy content. The distribution of radiated energy over wavelengths, as well as the total energy flux, depends on the temperature of the object. This can be approximated with a black-body, which is an idealized state of an object in thermodynamical equilibrium, where the object is a perfect absorber and emitter of radiation. That is, it absorbs all the radiation incident on it and emits the maximum possible amount of radiation. Based on both quantum mechanics and thermodynamics a black-body can be described 
by Planck's radiation law, where the wavelength distribution of radiation emitted from a black-body with temperature $T$ is given by:

$$
G_{b \lambda}=\frac{2 \pi h c^{2}}{\lambda^{5}\left(e^{\frac{h c}{\lambda k T}}-1\right)}
$$

where $k$ is Boltzmann's constant $\left(1.380 \times 10^{-23} \mathrm{JK}^{-1}\right) . G_{b \lambda}$ is often given in units of $\mathrm{Wm}^{-2} \mu \mathrm{m}^{-1}$. We can also express this distribution in terms of photon flux by dividing the emitted power at each wavelength by the corresponding photon energy:

$$
\Phi_{b \lambda}=\frac{\lambda}{h c} G_{b \lambda}
$$

in units of $\mathrm{s}^{-1} \mathrm{~m}^{-2} \mu \mathrm{m}^{-1}$. By differentiating the distribution in Equation 2.2 with respect to wavelength and equating to zero, the wavelength corresponding to the maximum of the distribution can be found. The relation between this wavelength and the black-body temperature is

$$
\lambda_{\max } T=2897.8 \mu \mathrm{mK}
$$

which is known as Wien's displacement law and states that the maximum wavelength is inversely proportional to the temperature. Another useful relation, which can be obtained by integrating Planck's law over all wavelengths, is the Stefan-Boltzmann equation. It expresses the total emitted black-body radiation:

$$
G_{b}=\int_{0}^{\infty} G_{b \lambda} d \lambda=\sigma T^{4}
$$

where $\sigma$ is the Stefan-Boltzmann constant $\left(5.670 \times 10^{-8} \mathrm{Wm}^{-2} \mathrm{~K}^{-4}\right) . G_{b}$ is in units of $\mathrm{Wm}^{-2}$. Note that this is also the unit most often used for incident solar radiation.

The latter two equations tell us something fundamental and familiar about heated objects: the Stefan-Boltzmann equation shows that the total radiated power increases with the temperature of the object and Wien's displacement law shows that the peak wavelength decreases with increasing temperature, which, for example, causes metals to glow brighter as they get hotter. 


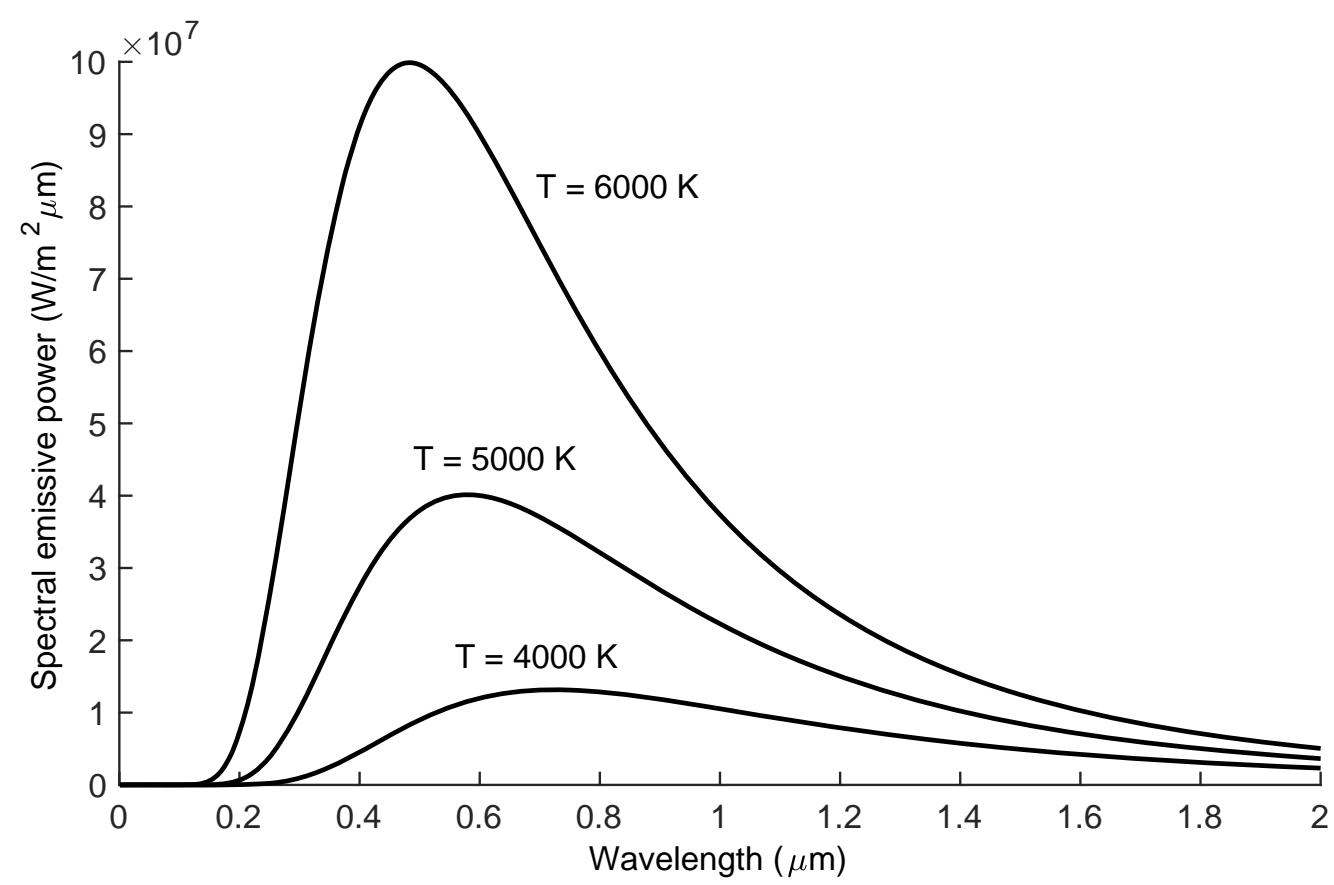

(a)

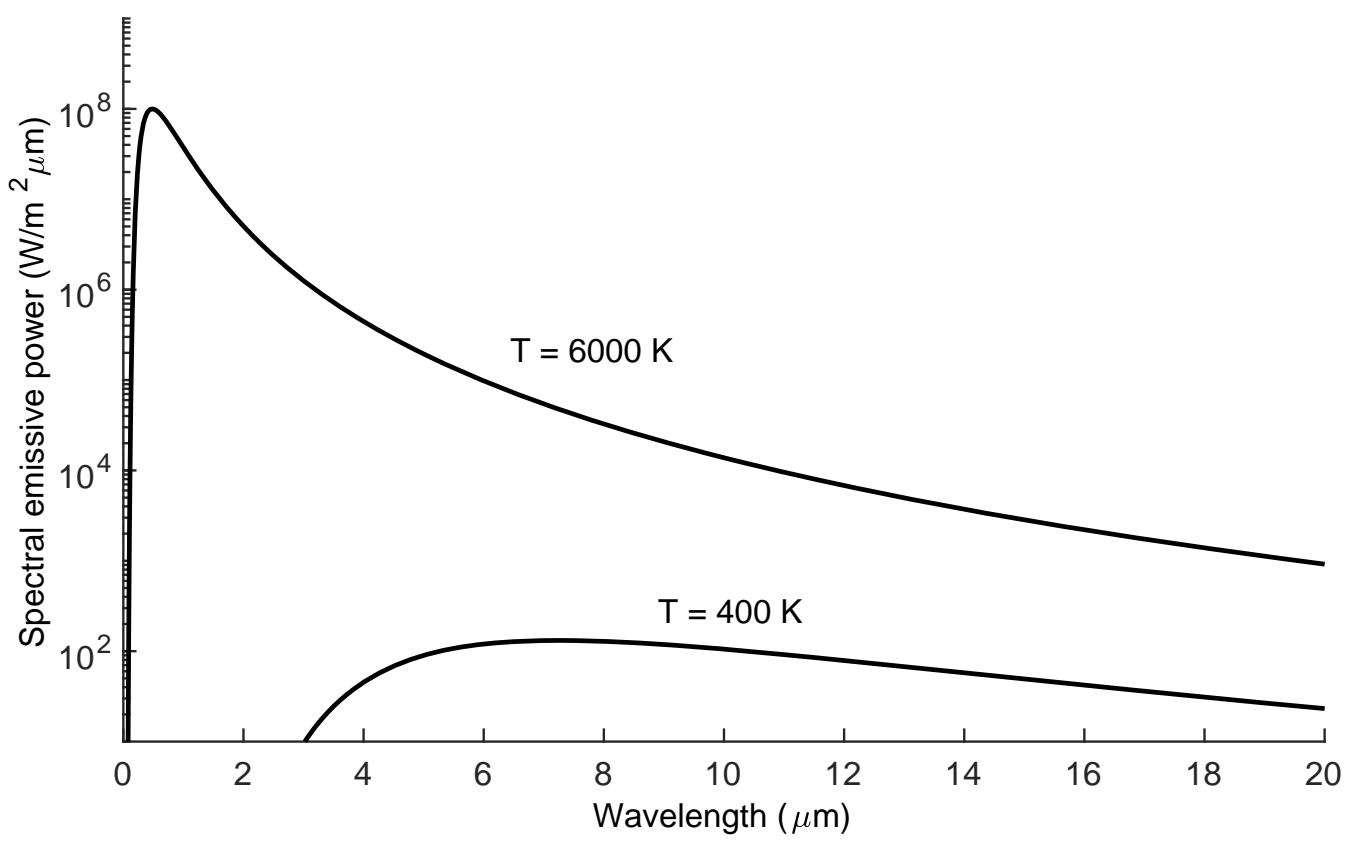

(b)

Figure 2.1: Wavelength distributions of electromagnetic radiation from black-bodies with different temperatures, determined from Equation 2.2. Note the logarithmic scale of the vertical axis in (b). 
These two properties of black-body radiation are shown in Figures 2.1(a) and 2.1(b). Both figures show the spectral distribution of the thermal radiation from a black-body with a temperature of $6000 \mathrm{~K}$ (approximately the surface temperature of the Sun). If the Sun were a true black-body, this is what we would expect its radiation distribution to look like. The figures also show black-body radiation curves at lower temperatures. Figure 2.1(b) (note the scale on the y-axis) clearly shows the difference between radiation from a black-body with a temperature of $6000 \mathrm{~K}$ and one with $400 \mathrm{~K}\left(127^{\circ} \mathrm{C}\right)$, which is closer to temperatures encountered in our daily environment.

\subsection{The solar constant}

Let us now turn to the actual solar radiation that reaches Earth's atmosphere. How much radiation is there and how is it distributed over the wavelengths? To start with, we note that solar radiation levels in the solar system drop with the square of the distance to the Sun. To realize this, assume that the Sun's radius is $r$ and its surface temperature $T$. The surface area of the Sun is then $4 \pi r^{2}$ and the total radiative flux from the Sun is, using the Stefan-Boltzmann equation, $\sigma T^{4} \times 4 \pi r^{2}$. Now, the surface of a larger imaginary sphere with radius $l$ with the Sun at its center will receive the same amount of radiation, but over the larger area $4 \pi l^{2}$. Consequently, the energy flux per unit area at the distance $l$ from the Sun is

$$
G_{l}=\frac{\sigma T^{4} \times 4 \pi r^{2}}{4 \pi l^{2}}=\sigma T^{4}\left(\frac{r}{l}\right)^{2} .
$$

If you use the black-body temperature of the Sun $(5777 \mathrm{~K})$, the radius of the Sun $\left(6.957 \times 10^{8} \mathrm{~m}\right)$ and the distance between the Sun and Earth $\left(1.495 \times 10^{11} \mathrm{~m}\right)$, you will get the average radiative flux just outside Earth's atmosphere, per unit area facing the Sun: $1367 \mathrm{~W} / \mathrm{m}^{2}$. This will be denoted by $G_{s c}$ and is called the solar constant, or the air mass zero (AM0) radiation.

$G_{s c}$ is not determined experimentally in this way but the other way around: it is the Sun's surface temperature that is inferred from measurements of $G_{s c}$ at Earth by using Equation 2.6 solved for the black-body 
temperature $T$. Estimates of $G_{s c}$ have gradually improved by radiation measurements outside the atmosphere using aircraft, ballons and spacecraft. The value $1367 \mathrm{~W} / \mathrm{m}^{2}$, adopted by the World Radiation Center (WRC), is commonly used in the solar engineering literature [2].

Through measurements it is also possible to determine the spectral distribution of the AM0 radiation. Figure 2.2 shows the standardized WRC spectral irradiance curve, obtained from high-altitude and space measurements. The spectral distribution of the AM0 radiation follows closely the shape of the black-body radiation curve that is obtained from evaluating Planck's law close to the surface temperature of the Sun (cf. Figure 2.1). The dissimilarities in the spectral distributions are due to absorption in the cooler, upper parts of the Sun's photosphere.

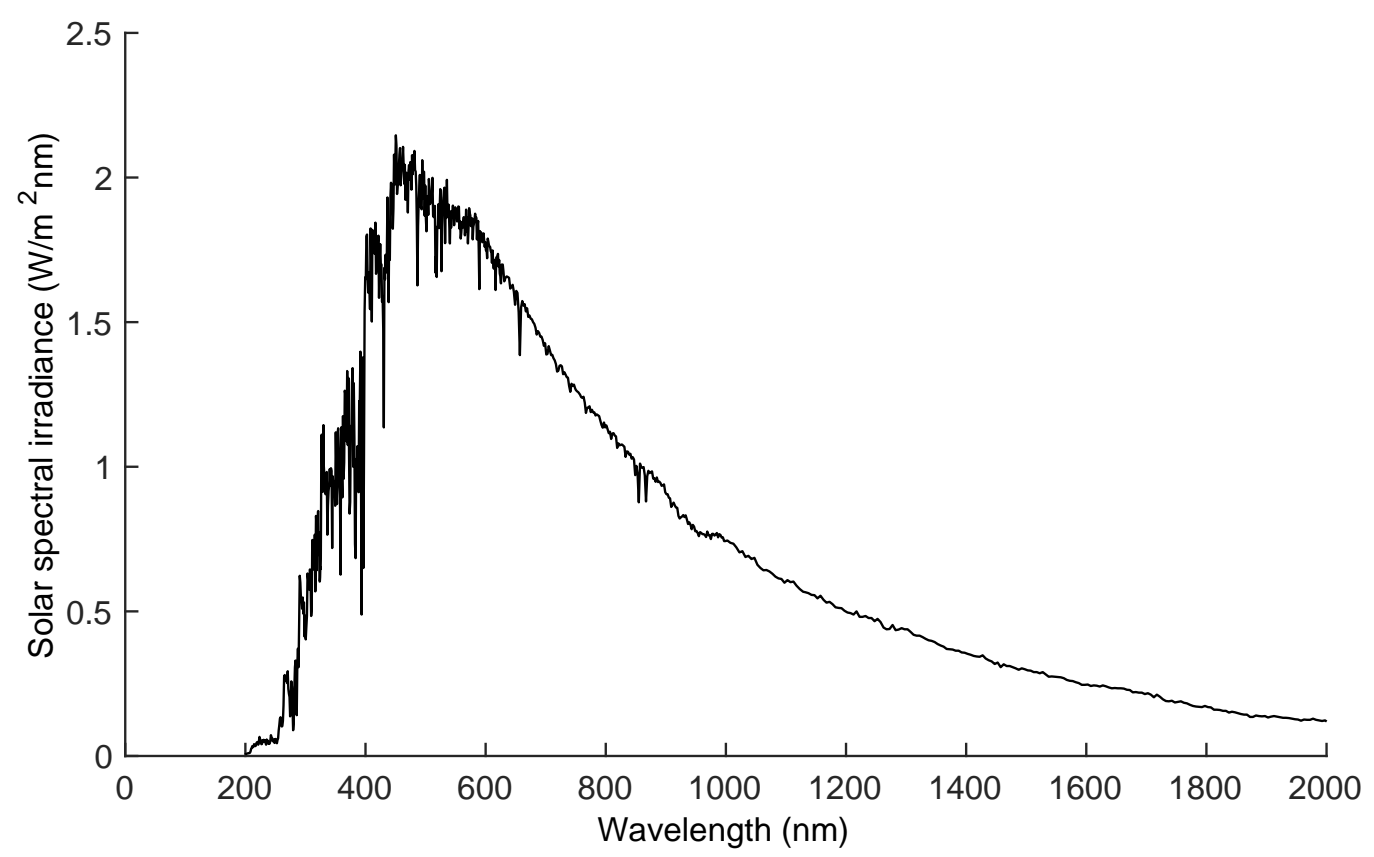

Figure 2.2: The standard WRC spectrum of AM0 radiation. Data obtained from NREL, USA [3].

Due to Earth's elliptic orbit around the Sun the actual solar radiation outside the atmosphere at a given time (the extraterrestrial radiation) will differ from $G_{s c}$. Over the year, it varies from $1412 \mathrm{~W} / \mathrm{m}^{2}$ at the beginning of July to $1322 \mathrm{~W} / \mathrm{m}^{2}$ at the turn of the year; a $3.3 \%$ variation 
from the mean value. This can be expressed mathematically as

$$
G_{0 n}=G_{s c}\left(1+0.033 \times \cos \left(360 \frac{d}{365}\right)\right)
$$

where $d$ is the day of the year. The subscript 0 denotes zero air mass (AM0) and the subscript $n$ indicates that the radiation is on a plane normal to the Sun-Earth axis.

\subsection{Categorization of radiation}

Based on properties and field of applications, electromagnetic radiation is categorized into wavelength bands. Figure 2.3 shows some common classifications in the ranges relevant for solar engineering purposes. The vast majority of solar radiation is in the wavelength range of approximately 0.3 to $3 \mu \mathrm{m}$ and spans parts of the ultraviolet (UV) and infrared (IR) ranges. A simpler classification of the wavelengths of importance in solar engineering is to divide the spectrum of solar and IR radiation into short-wave and long-wave radiation. Short-wave is the same as solar radiation and long-wave is everything with longer wavelengths. For the purpose of solar collectors it is a useful fact that the incident solar radiation in the short-wave range and the emitted radiation from solar collectors in the long-wave ranges do not overlap significantly. This is beneficial because it makes it possible to design materials and devices that have entirely different properties for short-wave solar and long-wave thermal radiation. 


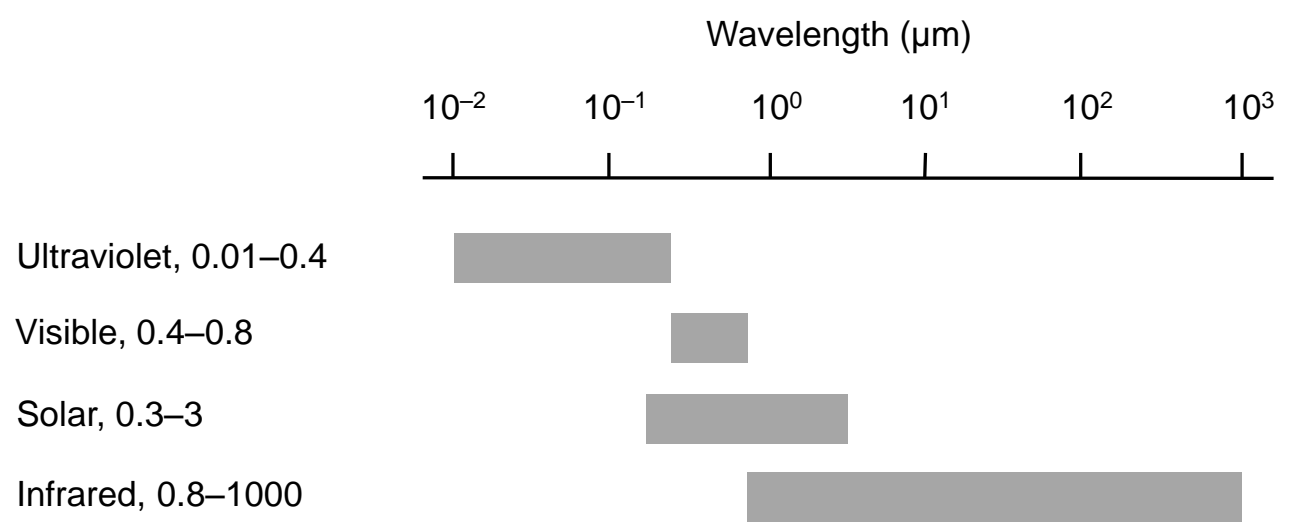

Figure 2.3: Some common wavelength bands in the spectrum of electromagnetic radiation. 


\section{Chapter 3}

\section{Available solar radiation on Earth}

Extraterrestrial radiation is affected in various ways during passage through the atmosphere. This section reviews these mechanisms and describes the properties of the solar radiation available at Earth's surface. A short discussion on the radiation and energy balance of Earth is also included.

\subsection{Atmospheric attenuation}

When passing through the atmosphere, solar radiation with normal incidence is subject to two sources of attenuation: scattering and absorption.

Scattering occurs when the radiation interacts with air molecules, water and dust in the atmosphere. The degree of scattering is determined by the wavelength of the radiation in relation to particle size, the concentration of particles in the atmosphere and the total mass of air that the radiation has to travel through. The most important process is Rayleigh scattering, in which light is scattered off air molecules. This type of scattering is most effective for shorter wavelengths in the blue end of the spectrum, mainly those shorter than $0.6 \mu \mathrm{m}$. This scattering process explains the blue color of the sky at daytime, the yellow color of the Sun and the reddening of the sky at night. This occurs because most of the radiation reaching the ground from other directions than directly from the Sun has been scattered by Rayleigh scattering. Note that a significant amount of the scattered light is redirected back into space. 
Absorption of solar radiation occurs in the UV range due to ozone and in the IR range due to water and carbon dioxide. In the process of absorption, the solar radiation is converted to heat, which is emitted by the particles as long-wave radiation.

The effect of Rayleigh scattering is quite large and wavelength-dependent, as can be seen in Figure 3.1.

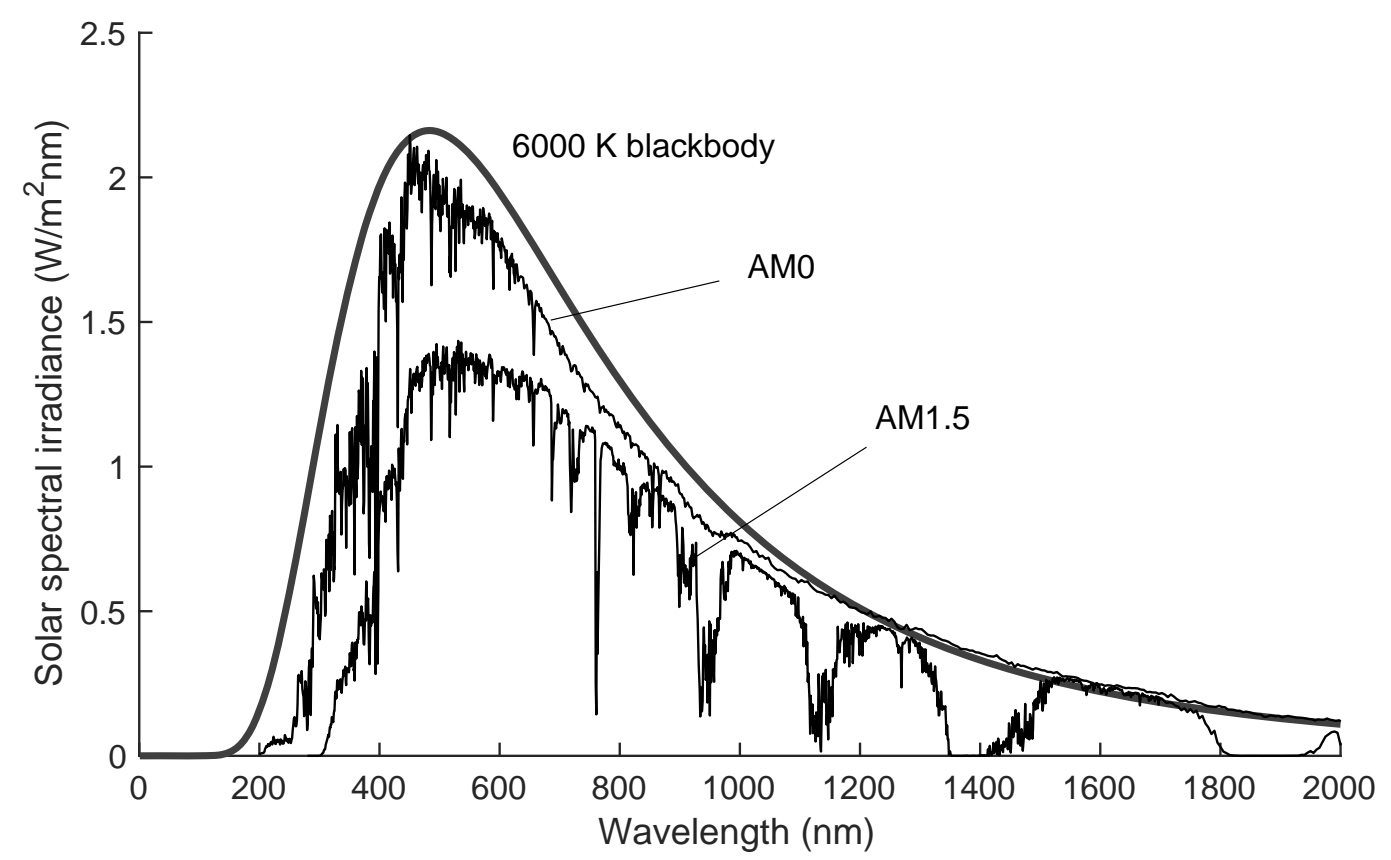

Figure 3.1: The AM1.5 spectrum in relation to the AM0 spectrum and the $6000 \mathrm{~K}$ blackbody radiation distribution weighted as in Equation 2.6. The drop in peak intensity at roughly $500 \mathrm{~nm}$ is due to Rayleigh scattering, the other dips are due to absorbtion from oxygen $\left(\mathrm{O}_{2}\right)$, ozone $\left(\mathrm{O}_{3}\right)$, water $\left(\mathrm{H}_{2} \mathrm{O}\right)$ and carbon dioxide $\left(\mathrm{CO}_{2}\right)$. The AM1.5 spectrum is specified by the National Renewable Energy Laboratory (NREL), USA [3].

\subsection{Air mass}

Attenuation of solar radiation depends on how far the radiation has to travel through the atmosphere. The longer the path length, the more particles the light has to interact with. This varies over the year and over individual days, with the longest path in the evenings, when the Sun is close to the horizon. The path length is described by the air mass. Formally, air mass is the ratio of the atmospheric mass through 
which the radiation passes from the Sun's current position in the sky, to the mass that it would pass through if the Sun were at the zenith (directly overhead). For example, at air mass 2, the path length through the atmosphere is two times longer than if the Sun were directly overhead. If the zenith angle, i.e. the angle from overhead to the Sun, is denoted by $\theta_{z}$, the air mass is to close approximation

$$
m=\frac{1}{\cos \theta_{z}}
$$

$\theta_{z}<70^{\circ}$ as shown in Figure 3.2. For higher angles the curvature of Earth becomes influential. For $\theta_{z}=85^{\circ}$ the error is $10 \%$. A more thorough discussion of air mass can be found in [4].

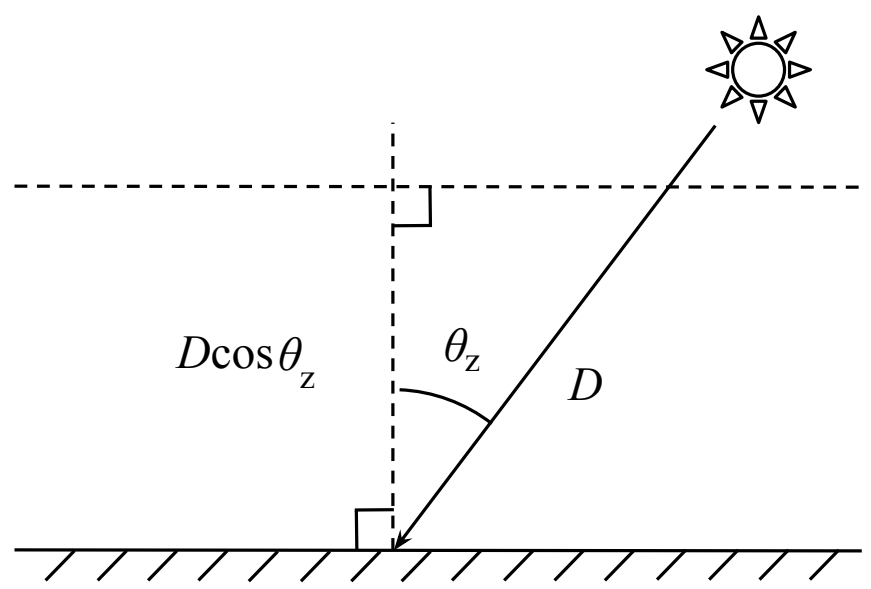

Figure 3.2: Atmospherical path length $D$ of solar radiation at zenith angle $\theta_{z}$.

Because the atmospheric conditions vary over time, a standard spectrum for radiation at ground level is needed for development and testing of solar devices. The accepted standard is the distribution for $m=1.5$, the AM1.5 spectrum, which corresponds to a zenith angle of $48.2^{\circ}$. Figure 3.1 shows the so-called AM1.5 spectrum and compares it to the extraterrestrial WRC spectrum and a $6000 \mathrm{~K}$ black-body distribution.

\subsection{Radiation components}

Because of scattering, radiation on Earth's surface consists of two components. Part of the incoming radiation is preserved as beam radiation, 
while the rest is scattered in the atmosphere and is either reflected back into space or reaches the ground as diffuse radiation. In contrast to beam radiation, which has a well-defined direction, diffuse radiation originates from all over the sky dome. Although diffuse radiation is most intense near the Sun, a good approximation is to assume that it is isotropic, i.e., uniformly distributed on all directions.

The proportions of diffuse and beam radiation are strongly dependent on weather conditions. In sunny weather with clear skies, some 10$20 \%$ of the radiation is diffuse. In cloudy weather with a lack of bright sunshine most of the incident radiation is diffuse. Figure 3.3 shows these proportions on two days in Norrköping, Sweden (59 35' $31^{\prime \prime}$ N $17^{\circ} 11^{\prime} 8^{\prime \prime}$ E). Over the year, the total diffuse part can be significant. For example, in Norrköping, between 1983 and 1998, the diffuse share of the total radiation was $51 \%$ on average. As a comparison, in one of Sweden's most sunny locations, Visby $\left(57^{\circ} 38^{\prime} 5^{\prime \prime} \mathrm{N} 18^{\circ} 17^{\prime} 57^{\prime \prime} \mathrm{E}\right)$, the diffuse part was still $47 \%$ for the same years [6].
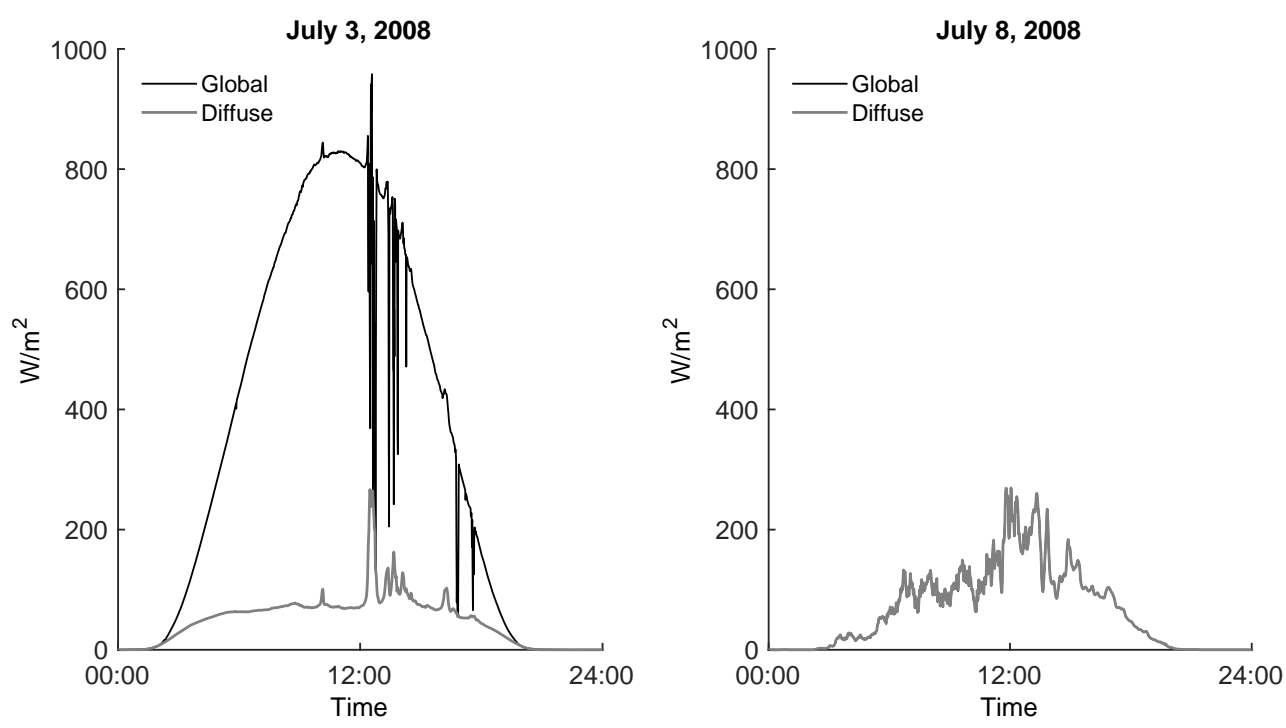

Figure 3.3: Global and diffuse radiation on the horizontal plane in Norrköping, Sweden, on two summer days in 2008, one clear day (left) with a few moving clouds in the afternoon and one completely overcast day (right). On the clear day the diffuse fraction was $14.2 \%$, on the overcast day it was $99.7 \%$. Note that the time here is UTC, i.e. Swedish standard time minus one hour (minus two hours including summer daylight savings time).

In addition, diffuse radiation has a different spectrum than beam radia- 
tion. The high-frequency part of the spectrum is more pronounced because Rayleigh scattering mainly affects shorter wavelengths. However, for most practical purposes the spectral distribution can be assumed to be the same as for beam radiation.

A third radiation component that has to be considered in solar energy applications is radiation reflected from the ground and from other surrounding objects onto a sloped surface. It depends on the reflectivity of the ground, the so-called albedo.

\subsection{Temporal and geographical variations in solar energy avail- ability}

Solar energy variability is fundamental to life on Earth as we know it. Due to Earth's tilted axis with respect to its orbit around the Sun, the solar energy availability varies over the year, giving rise to seasons. Because Earth rotates around its own axis, we have nights and days. Figure 3.4(a) shows Earth's tilt relative to the Sun at three particular points in time. On the summer solstice, the Northern Hemisphere is tilted maximally towards the Sun and experiences its longest day of the year. On the winter solstice, the Northern Hemisphere is tilted maximally away from the Sun and the day is the shortest of the year. Midway between the solstices are the equinoxes, where Earth's axis of rotation is perpendicular to the Sun and the Northern and Southern Hemispheres receive equal amounts of radiation.

The daily movement of the Sun across the sky for a latitude of $60^{\circ}$ $\mathrm{N}$, passing near by Norrköping, Sweden, on the same days is shown in Figure 3.4(b). At the summer solstice, the Sun reaches its highest position in the sky and at the winter solstice its lowest. Note also that at the equinoxes the Sun rises exactly in the east and sets exactly in the west $\left( \pm 90^{\circ}\right)$. During the summer half-year the Sun rises north of west (in the Northern Hemisphere) and sets north of east (if we are not above the Arctic Circle where the Sun sometimes does not set at all), and during winter it rises and sets south of east and west. How long the Sun is up and at which height (higher elevation means lower air mass) 
directly affects how much solar energy is maximally available each day. These seasonal and daily variations are completely predictable and form part of the mathematical framework outlined in Chapter 4.

These variations aside, there are also those caused by cloud movements and a varying turbidity (transparency) of the atmosphere. The shortterm irradiance variability that is caused by moving clouds can be considerable for a point location (cf. Figure 3.3), but over a larger area or a set of distributed surfaces the variations are smoothed out because all locations are not cloud-covered at the same time. Clear days, as well as completely overcast days, experience no significant short-term variability. Over the year, these stochastic variations tend to even out, but depending on the location there could be more consistent variations in cloudiness that may adversely affect certain seasons or parts of the day.

The solar radiation variability on Earth's surface due to cloudiness can more easily be quantified if the clear-sky irradiance is used to normalize it. This is called the clear-sky index and is defined as:

$$
\kappa \equiv \frac{G}{G_{c}}
$$

where $G$ is the global horizontal irradiance and $G_{c}$ is the clear-sky irradiance over some period of time. An example histogram of the clear-sky index based on nearly instantaneous (1-s) solar irradiance data from a pyranometer network on the Island of Oahu, Hawaii [5], and estimated clear-sky irradiance data (for solar elevation angles above 20 degrees to avoid effects of low solar elevations), is shown in Figure 3.5. The clearsky index distribution varies between locations, but typically has two pronounced peaks, one at around $\kappa=1$, which corresponds to bright sunshine, and one peak at around $\kappa=0.5$ which typically corresponds to overcast cloudiness. Values of $\kappa$ above 1 correspond to irradiance levels higher than for bright sunshine, and this occurs when sunlight is reflected off clouds. This effect is called cloud enhancement, which can also be seen in Figure 3.3 where there is a high peak exceeding the regular clear-sky pattern on the mainly clear day when clouds pass by.

Although the solar irradiance varies over time and location, there are measures which are preserved for each location over time when the radi- 
(a)

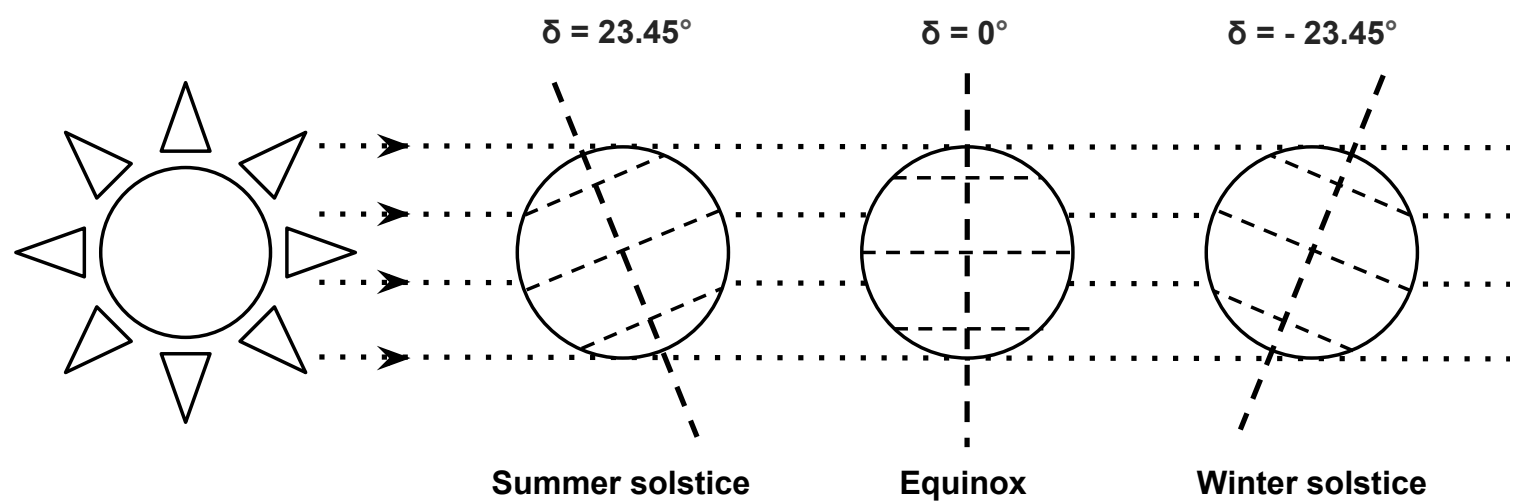

(b)

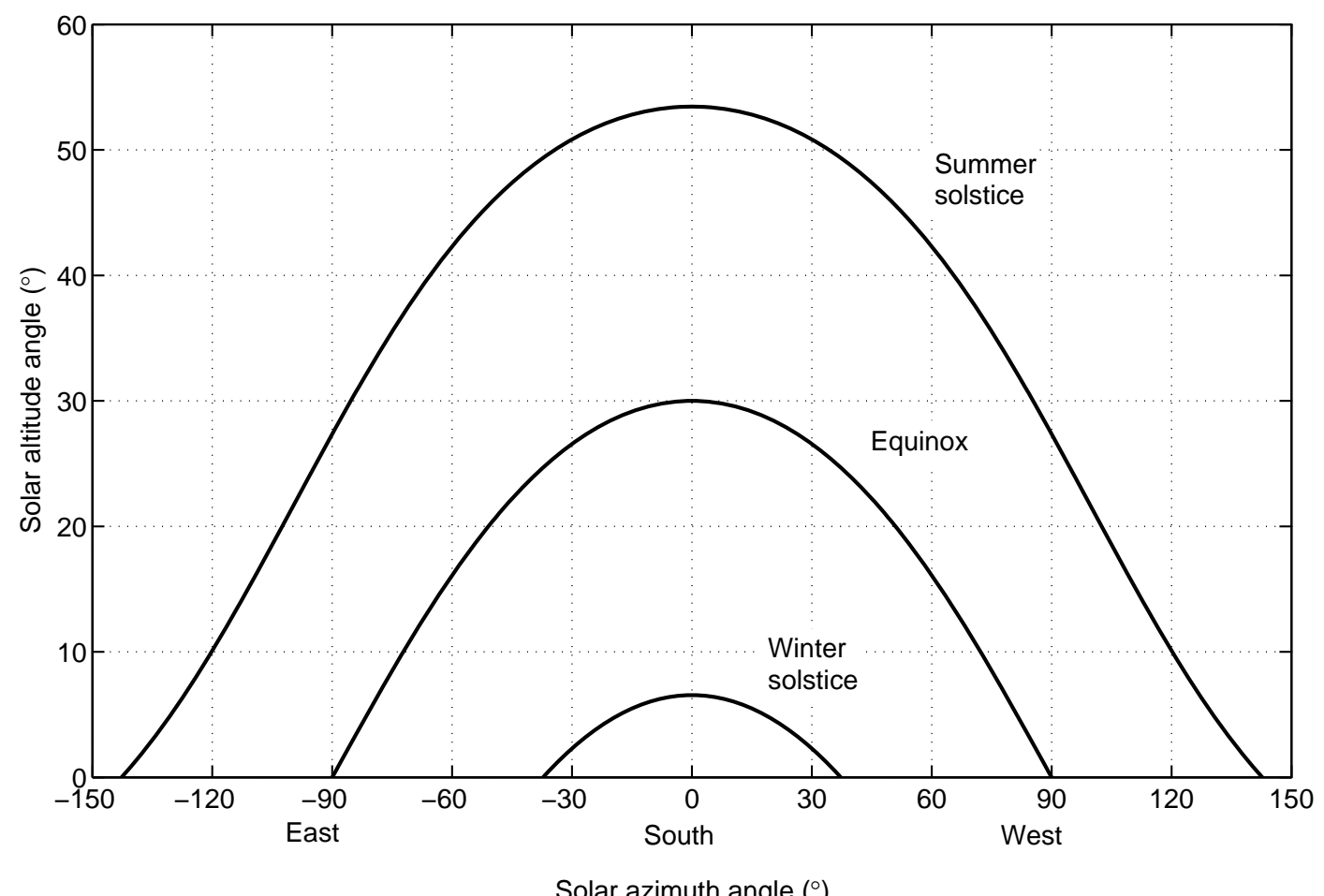

Figure 3.4: Seasonal and daily variations in Earth's tilt relative to the Sun and their impact on the solar altitude and azimuth. In (a) Earth's tilt with respect to the direction of incident solar radiation is shown at the summer solstice, the equinoxes and the winter solstice. The corresponding declination (the angle between the Sun's position and the equatorial plane) is shown. In (b) the corresponding Sun charts are shown at latitude $60^{\circ} \mathrm{N}$. The solar altitude angle is $90^{\circ}-\theta_{z}$. 


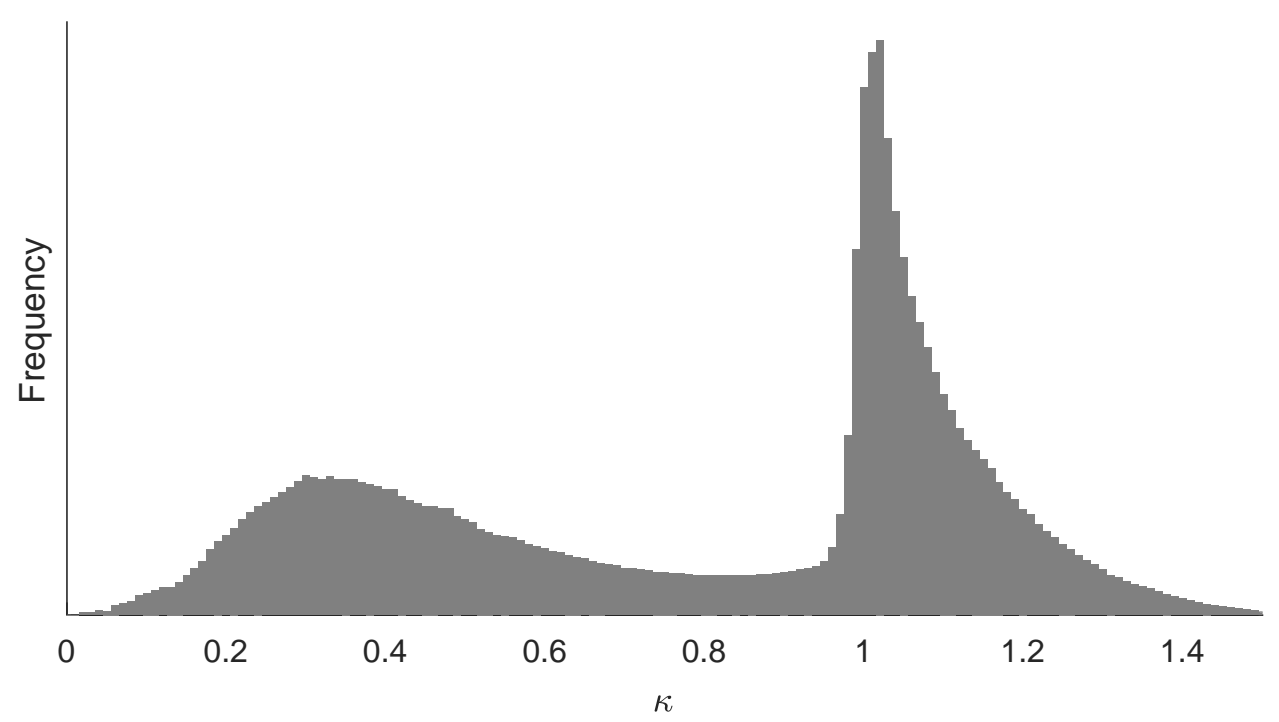

Figure 3.5: Histogram of the near-instantaneous clear-sky index, determined from 401 days 1-s resolution global horizontal irradiance measured in Oahu, Hawaii, in 2010 and $2011[5]$.

ation is averaged over a certain time period. A typical example of this is the Angström equation [2, p.64]:

$$
\frac{H}{H_{c}}=a+b S .
$$

This equation relates the total irradiation $H$ over a longer period (typically monthly), the total clear-sky irradiation $H_{c}$, and the fraction $S$ of number of bright sunshine hours relative to the total number of potential sunshine hours. In this equation $a$ and $b$ are set by local conditions and can be estimated by measuring $H$, calculating $H_{c}$ and obtaining a linear fit to $S$. For Stockholm, Sweden $\left(59^{\circ} 19^{\prime} 46^{\prime \prime} \mathrm{N} 18^{\circ} 4^{\prime} 7^{\prime \prime} \mathrm{E}\right)$, the parameters were estimated by Angström to $a=0.235$ and $b=0.765$ [7]. The equation is named after the meteorologist Anders Ångström (1888-1981), not to be confused with his grandfather the physicist and solar spectrum researcher Anders Jonas Ångström (1814-1874) [9].

The Sun's movements across the sky in combination with the local climate, weather and terrain conditions make the solar resource vary geographically. There is a consistent variation with the latitude, with less energy available in the north and more in the south, but also latitude- 
independent variations that are due to weather and terrain. In general, more solar energy is available in coastal regions and less in mountainous areas.

\subsection{Earth's energy balance}

Solar radiation is the most important source of energy on the planet. Other sources include geothermal energy and tidal energy resulting from the moon's gravitational pull, but the flux of solar energy is very high in comparison. Around 130000 Gtoe (toe $=$ tonnes of oil equivalents) reach Earth every year, while the contribution from geothermal energy from Earth's mantle is 19 Gtoe and from tidal energy 2 Gtoe [8].

An intricate energy balance between incoming and outgoing radiation is maintained on Earth. Almost one third of the incident solar energy is reflected back into space, while the rest is absorbed in the atmosphere, by oceans and in inorganic and organic matter on Earth. The absorbed solar energy drives meteorological and hydrological processes such as winds, waves and ice melting, and it fuels photosynthesis in the biosphere. The total radiation balance is maintained by outgoing long-wave radiation emitted from Earth's surface. Part of this radiation leaves Earth while a substantial part is re-radiated back to Earth. This cycle of surface radiation and back-radiation by the atmosphere is maintained by the greenhouse gases. A simplified outline of this complex balance is summarized in Figure 3.6.

Atmospheric damping by absorption influences both solar radiation reaching Earth and thermal emission from the ground. Most solar energy can be transmitted to ground level, due to a gap in the atmospheric absorption, with the exception of the UV and IR parts of the solar spectrum, which are strongly damped (cf. Figure 3.2). Thermal radiation is absorbed by the atmosphere, except in the $8-13 \mu \mathrm{m}$ range. This is the so-called atmospheric window (cf. Figure 3.6), which is the main channel for loss of gained energy from Earth's surface. Absorption in this part of the spectrum is dominated by $\mathrm{CO}_{2}$ and $\mathrm{H}_{2} \mathrm{O}$. Consequently, increased concentrations of greenhouse gases in the atmosphere cause the atmo- 
spheric window to narrow, which is the main issue of global warming. The main point with this short account is that ambient radiation and natural systems' response to it are spectrally selective, which is crucial and fundamental to life on Earth, but also something that can be utilized in engineering of solar devices. A more thorough discussion can be found in $[12]$.

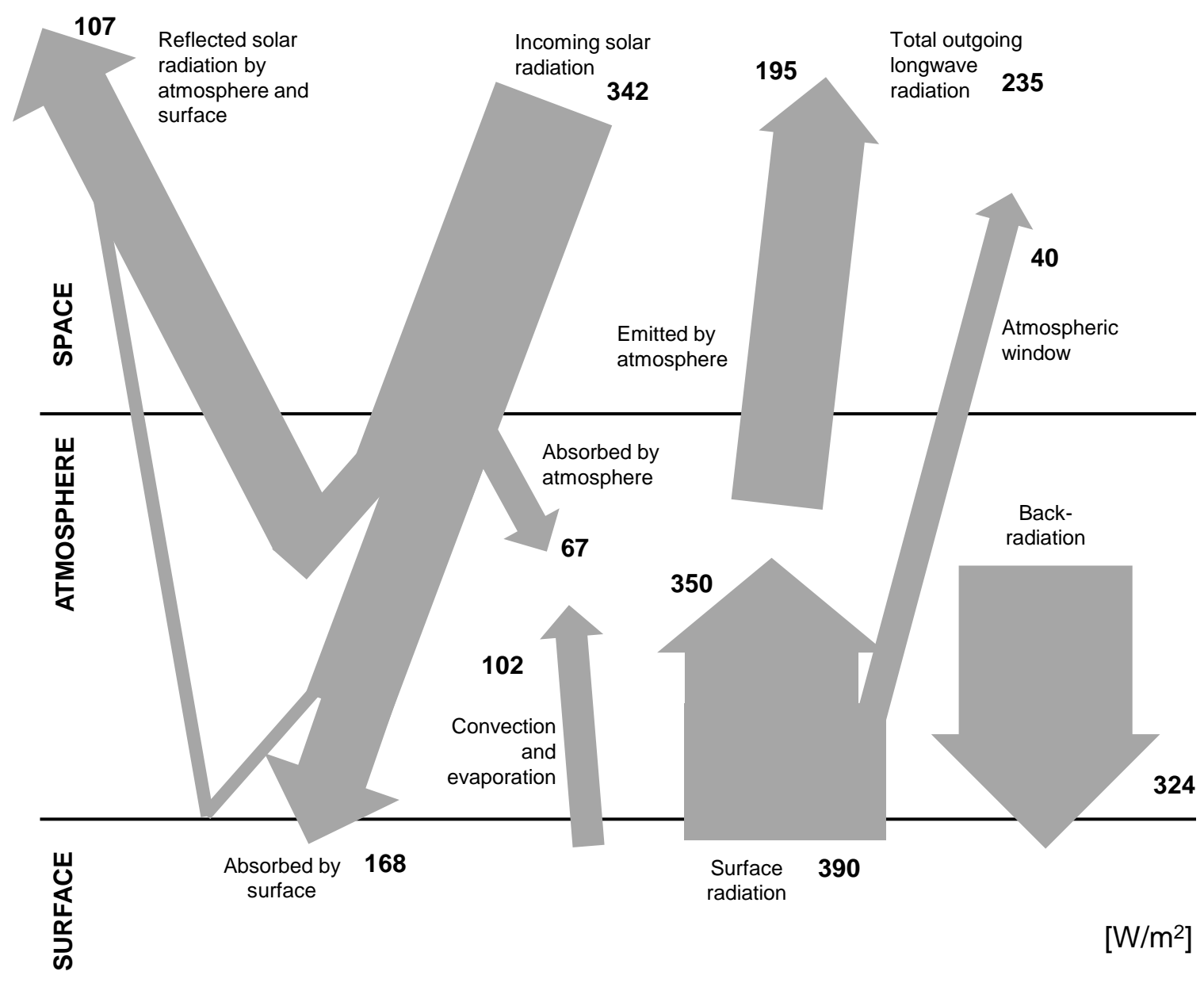

Figure 3.6: The radiation and energy balance of Earth. Based on [11]. 


\section{Chapter 4}

\section{Quantifying available solar energy on planar surfaces}

Solar energy systems typically have a receiving surface that collects solar radiation, e.g., a PV array or a solar collector. In order to optimize the orientation of this surface, we must be able to estimate how much solar energy differently oriented surfaces collect. This is complex because the Sun is moving across the sky and seldom faces a fixed surface directly, and because the different radiation components (beam, diffuse and ground-reflected) reach the surface from different angles. Varying weather conditions also make the availability of these different components differ from one time to another. For a reliable yield calculation, we must be able to convert commonly available hourly solar radiation data on the horizontal plane to radiation on a sloped, planar surface.

The aim of the following sections is to provide a mathematical framework for converting solar radiation measured on the horizontal plane to radiation on an arbitrarily oriented planar surface. This set of equations is often used in professional software for simulation and optimization of solar thermal or photovoltaic systems based on measured horizontal irradiance. It is also very useful for understanding the important parameters that influence collection of solar energy on a surface. These and other radiation formulae and mathematical models are discussed in more depth in $[2]$. 
28 Chapter 4. Quantifying available solar energy on planar surfaces

\subsection{Measured solar irradiance data}

Determination of solar irradiance on tilted surfaces typically starts from measurements in the horizontal plane. Solar radiation is commonly measured by two main classes of instruments: pyrheliometers and pyranometers. A pyrheliometer measures solar radiation coming directly from the Sun and a small portion of the sky around the Sun at normal incidence. In this device sunlight typically enters through a window to a thermopile (a device that converts heat to electricity). The electrical signal that is generated can be recorded and converted into $\mathrm{W} / \mathrm{m}^{2}$. The window of the pyrheliometer acts as a filter that only lets through sunlight in the 0.3-3 $\mu \mathrm{m}$ range.

The pyranometer measures total hemispherical (diffuse plus beam) solar radiation, usually on the horizontal plane. This means that the device must give an unbiased response to radiation from all directions. It consists of a thermopile sensor that is horizontally oriented and a glass dome that limits the wavelength range, as in the pyrheliometer. The glass dome preserves the $180^{\circ}$ view and shields the thermopile from air convection. Schematic illustrations of a pyranometer and a pyrheliometer are shown in Figure 4.1.

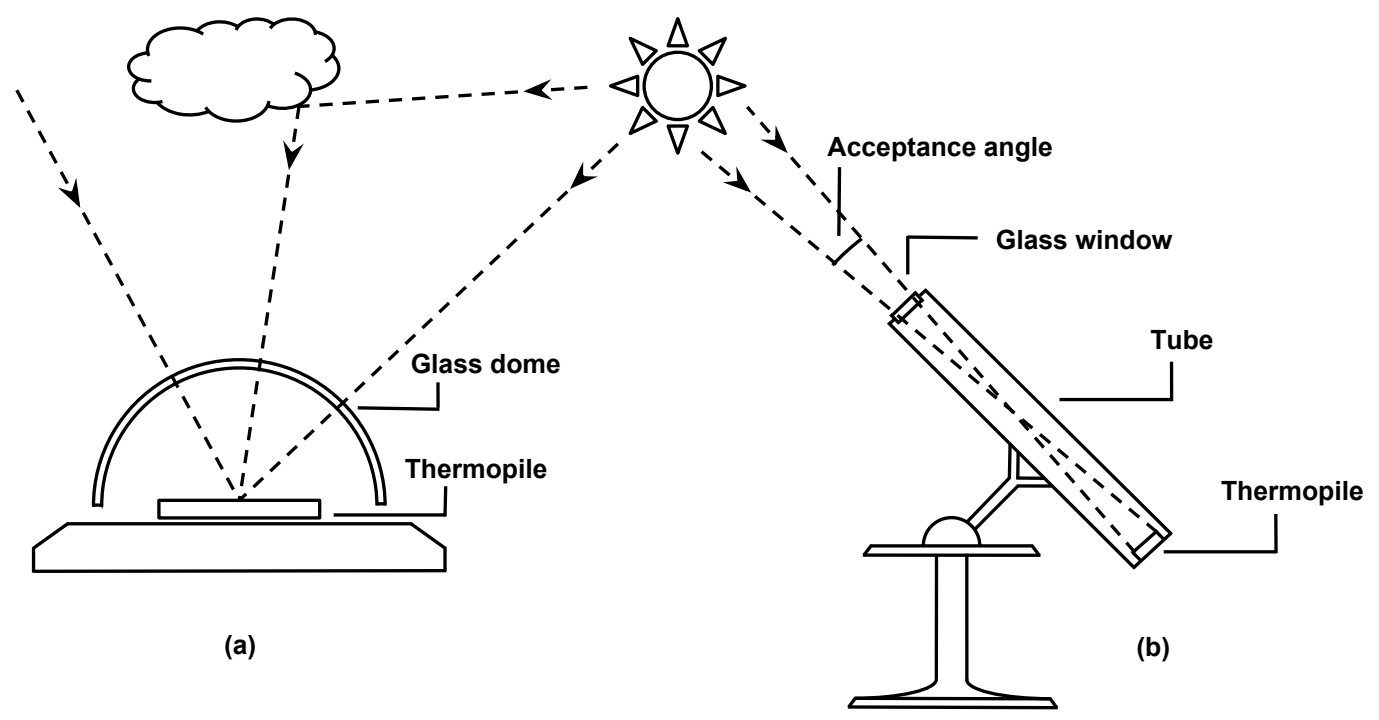

Figure 4.1: A schematic illustration of a pyranometer (a) and a pyrheliometer (b), based on [9] and [10]. 
While pyrheliometers only measure normal-incidence beam radiation, global or diffuse radiation can be measured with pyranometers. To measure diffuse radiation a shading ring is attached to the pyranometer to block beam radiation. A correction factor is then used to compensate for the loss of view. Beam radiation on the horizontal surface can indirectly be measured by two pyranometers, one measuring diffuse and one measuring global, and then be obtained as the difference between global radiation and diffuse radiation.

In the following, we will deal with average radiative flux over some period of time, typically one hour, which is how monitored data are available. This means that the available radiation is integrated over both time and wavelengths. We will denote integrated and averaged radiation by the letter $I$ :

$$
I=\frac{1}{t_{2}-t_{1}} \int_{t_{1}}^{t_{2}} G(t) d t .
$$

Monitored radiation is available in time series, and the equations below are all applied one time step at a time. The time $t$ always refers to the midpoint of the monitored time interval:

$$
t=\frac{t_{1}+t_{2}}{2} .
$$

We will assume that we have the beam and diffuse radiation components $I_{b}$ and $I_{d}$ on the horizontal plane. From these we want to obtain the beam, diffuse and ground-reflected radiation components $I_{b T}, I_{d T}$ and $I_{g T}$ on a tilted planar surface. Expressed in global radiation we wish to go from radiation on the horizontal plane:

$$
I=I_{b}+I_{d}
$$

to radiation on the tilted plane:

$$
I_{T}=I_{b T}+I_{d T}+I_{g T} .
$$

The basic strategy is to find the incidence angles of radiation on the horizontal and tilted surfaces, which are used to weight beam radiation, and the view factor of the tilted plane, which determines the incident isotropic radiation on the tilted plane. 
Chapter 4. Quantifying available solar energy on planar surfaces

\subsection{Solar time}

In all of the following equations we use solar time, which is different from standard clock time. In fact, the Sun is a bad time-keeper, at least when precision within the hour is required. This is because the Sun's apparent movement across the sky is not uniform. Rather, since Earth's orbit is elliptic and Earth is tilted with respect to the orbit, the Sun appears to speed up and slow down over the year, sometimes being ahead of a regular clock, sometimes behind, up to as much as 16 minutes. This can be corrected for by a factor called the equation of time. On top of this, standard clock time is set uniform across individual time zones. These zones vary in width, but the widest can take the Sun several hours to cross, which gives substantial differences between standard and solar time for locations far from the standard meridians. Correcting for all of this, the solar time is, in minutes,

$$
t_{s}=t_{s t}-4\left(L_{s t}-L_{l o c}\right)+E(d),
$$

where $t_{s t}$ is the standard time in minutes after midnight, $L_{s t}$ is the standard meridian and $L_{l o c}$ is the longitude of the location. The second term makes use of the fact that the Sun transverses $1^{\circ}$ of longitude in four minutes. We have to be careful with the sign in front of this term, which here holds for longitudes counted positive east. $E(d)$ is the equation of time for day $d$ of the year, in an empirical formula from [4]:

$$
\begin{aligned}
E(d)=229.18(0.000075+ & 0.001868 \cos B-0.032077 \sin B \\
& -0.014615 \cos 2 B-0.04089 \sin 2 B)
\end{aligned}
$$

where

$$
B=(d-1) \frac{360}{365}
$$

Note that daylight savings time introduces yet another one-hour difference between standard clock time and solar time that has to be corrected for. 


\subsection{Solar angles}

In order to make conversions between horizontal and tilted planes, it is necessary to know the geometric relationships between the planes and between the planes and the Sun at any instant of time. A set of angles, defined below and shown in Figure 4.2, are used to define these relationships. First, we have the constant angles defining the orientation and location of the tilted plane:

- $\beta$, tilt of the plane with respect to the horizontal, $0^{\circ} \leq \beta \leq 180^{\circ}$

- $\gamma$, azimuth angle of the tilted plane, zero due south, west positive, $-180^{\circ} \leq \gamma \leq 180^{\circ}$

- $\phi$, latitude of the location, north positive, $-90^{\circ} \leq \phi \leq 90^{\circ}$

Second, we have the two time-varying angles that define the position of the Sun relative to the celestial sphere ${ }^{1}$ and Earth:

- $\delta$, declination of the Sun, the "vertical" position of the Sun on the celestial sphere, measured in degrees above or below the celestial equator, north positive $-23.45^{\circ} \leq \delta \leq 23.45^{\circ}$

- $\omega$, hour angle, the angular displacement of the Sun relative to the local meridian, zero at noon, afternoon positive, $-180^{\circ} \leq \omega \leq 180^{\circ}$

The declination makes a complete cycle in one year and can therefore be modeled with reasonable accuracy as a function of the day of the year $d$ :

$$
\delta=23.45 \sin \left(360 \frac{284+d}{365}\right) .
$$

The hour angle, which is the measure of time in the equations, is determined from the time of the day. It makes a complete cycle over 24

\footnotetext{
${ }^{1}$ The celestial sphere is an imaginary sphere surrounding Earth, on which all celestial objects are projected. When Earth moves around the Sun, it views the Sun against this backdrop of surrounding space. From the viewpoint of Earth, the Sun continuously traverses the celestial sphere, making a complete path across it in one year. This path is called the ecliptic. The celestial equator is the projection of Earth's equator on the celestial sphere.
} 
(a)

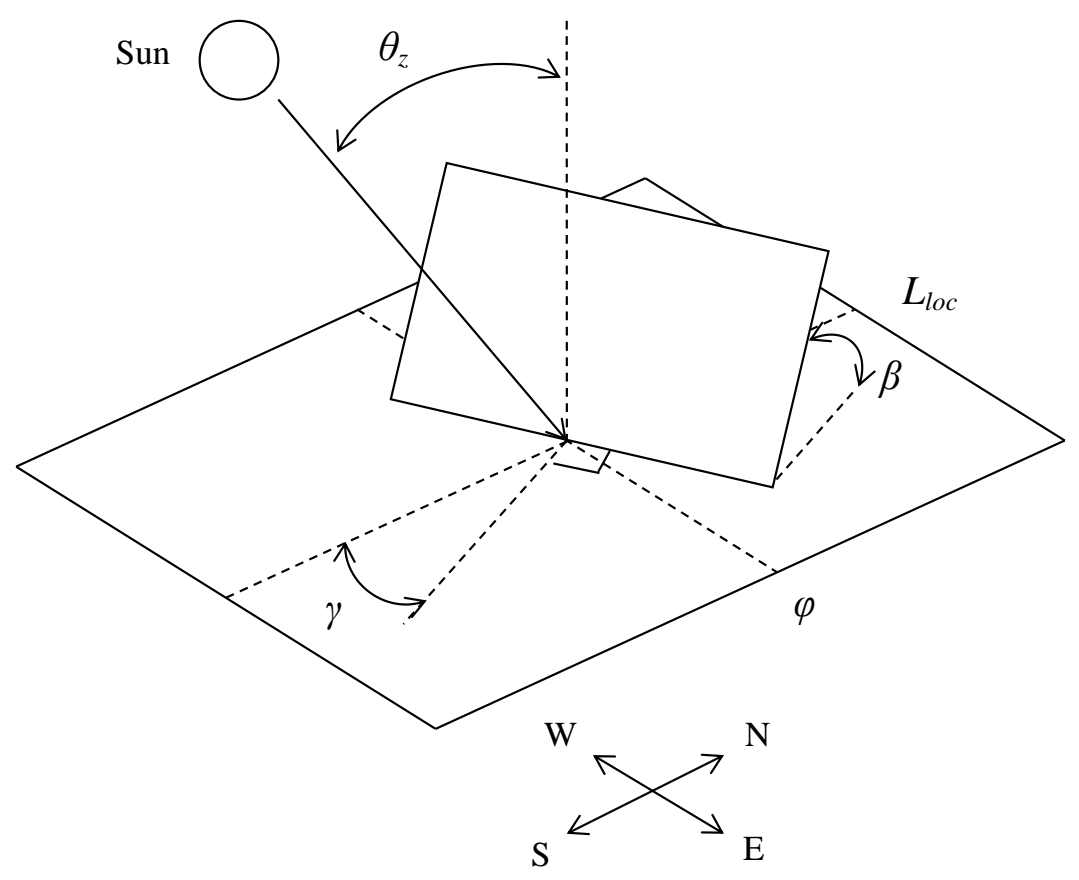

(b)

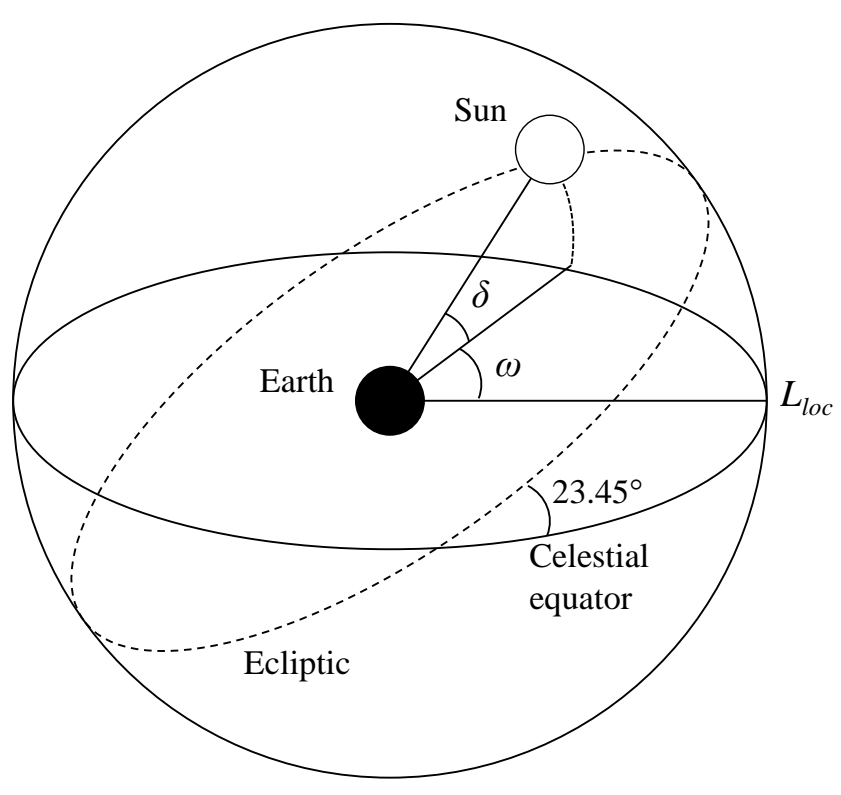

Figure 4.2: Solar angles used in the calculations. In (a) the angles that define the location and orientation of the tilted plane are shown. These are the plane tilt $\beta$, the azimuth angle $\gamma$ and the latitude $\phi$. Also shown are the longitude $L_{l o c}$ and the zenith angle $\theta_{z}$. In (b) the angles defining the Sun's position relative to Earth and the celestial sphere are shown; the declination $\delta$ and the hour angle $\omega$. 
hours. Since the Sun is displaced $15^{\circ}$ westward each hour due to rotation of Earth around its own axis and is zero at solar noon, the hour angle is

$$
\omega=15\left(\frac{t_{s}}{60}-12\right)
$$

where $t_{s}$ is the solar time of the day in minutes.

From these angles, we can find:

- $\theta$, angle of incidence, the angle between the normal to the tilted plane and the beam radiation on that surface, $-180^{\circ} \leq \theta \leq 180^{\circ}$.

The angle of incidence is related to all the other angles according to the following equation:

$$
\begin{aligned}
\cos \theta= & \sin \delta \sin \phi \cos \beta \\
& -\sin \delta \cos \phi \sin \beta \cos \gamma \\
& +\cos \delta \cos \phi \cos \beta \cos \omega \\
& +\cos \delta \sin \phi \sin \beta \cos \gamma \cos \omega \\
& +\cos \delta \sin \beta \sin \gamma \sin \omega .
\end{aligned}
$$

For a horizontal plane, the plane tilt $\beta=0$, and the above relationship is simplified accordingly. The resulting angle of incidence $\theta_{z}$, the zenith angle, which we have already encountered in the discussion on air mass, satisfies

$$
\cos \theta_{z}=\cos \phi \cos \delta \cos \omega+\sin \phi \sin \delta
$$

For a derivation of these equations for $\theta$ and $\theta_{z}$, see Appendix A.

\subsection{Extraterrestrial radiation}

For many radiation calculations it is useful to know the extraterrestrial radiation on the horizontal plane. We have already seen that extraterrestrial radiation with normal incidence can be expressed by Equation 2.7. To obtain the radiation on the horizontal plane, simply multiply by the cosine of the zenith angle:

$$
G_{0}=G_{s c}\left(1+0.033 \cos \frac{360 d}{365}\right) \cos \theta_{z} .
$$


An explanation of why this weighting provides the radiation on the horizontal plane is given in Figure 4.3. To obtain the average extraterrestrial radiation in a whole time step we would have to integrate this between the endpoints of the interval. However, as a good approximation we will assume

$$
I_{0} \approx G_{0}
$$

which means we pick the instantaneous value for the midpoint of the time interval.

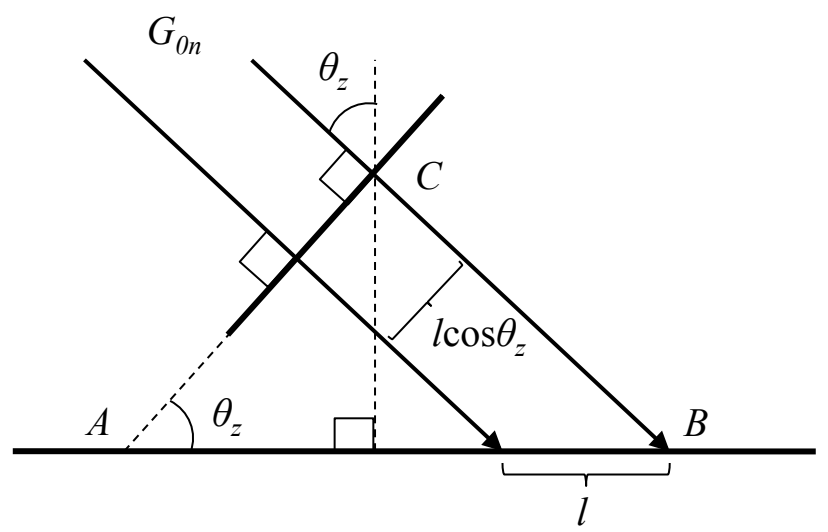

Figure 4.3: Conversion between extraterrestrial radiation with normal incidence and extraterrestrial radiation on the horizontal plane. In the triangle $A B C$, defined by the plane perpendicular to the radiation, the horizontal plane and the path of the radiation, the length of side $\mathrm{AC}$ is a factor $\cos \theta_{z}$ shorter than the side $\mathrm{AB}$. This holds also for sections of the triangle sides defined by parallel rays of radiation (as indicated by the sections with lengths $l$ and $l \cos \theta_{z}$ ). Consequently, the same amount of radiation incident on a unit area on the normal plane will reach an area $1 / \cos \theta_{z}$ on the horizontal plane, which means the radiation on the horizontal plane is a factor $\cos \theta_{z}$ less intense. Note that this relation holds for all beam radiation and all planes.

\subsection{Beam radiation on tilted surfaces}

When converting beam radiation between planes we use the geometric factor $R_{b}$, which is defined as the ratio of beam radiation on the tilted plane to beam radiation on the horizontal plane:

$$
R_{b}=\frac{I_{b T}}{I_{b}}
$$


Following the same reasoning as in Figure 4.3, we find that if $I_{b n}$ denotes the beam radiation on a plane perpendicular to the incident radiation, then $I_{b T}=I_{b n} \cos \theta$ and $I_{b}=I_{b n} \cos \theta_{z}$, and consequently,

$$
R_{b}=\frac{I_{b n} \cos \theta}{I_{b n} \cos \theta_{z}}=\frac{\cos \theta}{\cos \theta_{z}} .
$$

We can then express beam radiation on the tilted plane as

$$
I_{b T}=R_{b} I_{b}
$$

An $R_{b}$ value evaluated at the midpoint of a time interval is used as representative of the whole interval. As mentioned in [2], this is often a good-enough procedure for hourly values. The conversion is only performed when the Sun is above the horizon. Thus, $R_{b}$ is defined as above when both $\cos \theta>0$ and $\cos \theta_{z}>0$, and is zero otherwise. One thing to be cautious about is the risk of getting unrepresentative values of $R_{b}$ on intervals involving sunrise and sunset when $\cos \theta_{z}$ is close to zero, which may cause highly unrealistic morning or evening radiation peaks.

\subsection{Diffuse radiation on tilted surfaces}

Now that we can handle beam radiation, we need some similar weighting for scattered diffuse radiation. If we assume that the diffuse radiation from the sky is purely isotropic, this weighting factor is:

$$
f_{\text {view }, \text { sky }}=\frac{1+\cos \beta}{2}
$$

which is the sky view factor of the surface and describes how much of the sky is visible to the surface. It is easy to see that this expression makes sense, noting, e.g., that $f_{\text {view,sky }}=1$ when the surface is horizontal, $f_{\text {view }, \text { sky }}=\frac{1}{2}$ when it is tilted by $90^{\circ}$ and $f_{\text {view, sky }}=0$ when it is facing the ground. Analogously, the view factor to isotropic radiation from the ground can be found as

$$
f_{\text {view,ground }}=\frac{1-\cos \beta}{2} .
$$


The diffuse radiation component is normally considered to consist of three parts - isotropic diffuse, circumsolar diffuse and horizon brightening - which differ in their origin in the sky. The isotropic diffuse part is uniform from every direction, the circumsolar diffuse part is concentrated around the Sun's position in the sky and horizon brightening is concentrated near the horizon. Different models have been formulated to describe diffuse radiation on the tilted plane. For this compendium the so-called Hay and Davies model was chosen. Besides treating part of the diffuse radiation as isotropic, it also models circumsolar radiation. As has been shown in [13], the Hay and Davies model performs similarly to other, more complex models.

In the Hay and Davies model, diffuse radiation on the tilted surface is expressed as

$$
I_{d T}=I_{d}\left[\left(1-A_{i}\right)\left(\frac{1+\cos \beta}{2}\right)+A_{i} R_{b}\right]
$$

where the anisotropy index $A_{i}$ is defined as the ratio between the incident beam radiation $I_{b}$ and the extraterrestrial radiation $I_{0}$ on the horizontal plane:

$$
A_{i}=\frac{I_{b}}{I_{0}}
$$

Thus, since $I_{0}$ is the incident radiation that would be theoretically possible if there was no atmosphere, $A_{i}$ is the fraction of radiation that is preserved as beam radiation after it has passed through the atmosphere. Under clear conditions, $I_{b}$ approaches $I_{0}$, causing $A_{i}$ to be close to 1 , and the diffuse is treated in the same way as beam radiation:

$$
I_{d T} \approx I_{d} R_{b}
$$

When there is no beam radiation, $A_{i}$ is zero, and the diffuse radiation is considered purely isotropic:

$$
I_{d T}=I_{d}\left(\frac{1+\cos \beta}{2}\right)
$$

where $I_{d}$ is modified only by the view factor to the sky. 


\subsection{Ground-reflected radiation}

The third and last component of the total radiation on the tilted plane is the radiation reflected from the ground. In reality, numerous objects such as buildings, different ground materials, trees, etc., reflect incident radiation onto the tilted surface. A simplified but standard approach is to assume reflected radiation from one composite source, a horizontal, diffusely reflecting ground. The ground-reflected radiation on the tilted plane is then dependent only on the reflectance of the ground and the view factor to the ground of the tilted surface:

$$
I_{g T}=I \rho_{g}\left(\frac{1-\cos \beta}{2}\right)
$$

where $\rho_{g}$ is the ground reflectance and $I=I_{b}+I_{d}$ is the global radiation on the horizontal plane. The ground reflectance $\rho_{g}$ depends on the surroundings. At high latitudes, a seasonal variation in ground reflectance is likely because of snow coverage in the winter.

\subsection{Complete model for tilted plane global radiation}

The complete model for global radiation on a tilted plane is:

$$
I_{T}=I_{b T}+I_{d T}+I_{g T}
$$

With the formulae for each component in Equations 4.16, 4.22 and 4.23 the complete model is:

$$
\begin{array}{r}
I_{T}=I_{b} R_{b}+I_{d}\left[\left(1-A_{i}\right)\left(\frac{1+\cos \beta}{2}\right)+A_{i} R_{b}\right]+ \\
+\left(I_{b}+I_{d}\right) \rho_{g}\left(\frac{1-\cos \beta}{2}\right) .
\end{array}
$$

\subsection{Some notes on optimization of surface orientation}

Some general rules of thumb can be drawn up about how a surface should be tilted to maximize collection of solar radiation over a certain period of 
time. Further details can be found, e.g., in [14]. In general, the surface should be oriented so that the incidence angle $\theta$ is as close to zero as possible, as often as possible. Only a two-axis tracking system can keep the incidence angle at zero at all times. For a fixed surface we have to find an orientation that is suboptimal on most occasions but as good as possible over a longer period of time.

The azimuth angle $\gamma$ should be chosen so that the surface catches the Sun when it is at its highest point on the sky. On the northern hemisphere the surface should be directed south and on the southern hemisphere north. Exceptions include if there is some object shading the Sun (if there is shading in the east, the surface should be oriented west and vice versa), if there are systematic climatic conditions favoring morning or evening sun or if the collection should match some specific demand profile with peaks in the morning or evening (which applies mainly to off-grid PV systems).

The surface tilt $\beta$ depends on which season the collection is optimized for. Summer collection favors lower tilt angles than annual collection and winter collection requires even higher tilts. For sites within $30^{\circ}$ of the equator annual energy collection is optimized when the tilt is set roughly equal to the latitude. At higher latitudes lower tilt angles are more favorable in summer because more energy is available in the summer than at lower latitudes. A high albedo further favors high tilt angles because it is better with a higher view factor to the ground. 


\section{Chapter 5}

\section{Exercises}

\section{Check your understanding}

These questions cover the most important concepts. All answers are found in the sections above.

1. Describe, qualitatively, how the radiation emitted from a black-body changes when its temperature increases. How are the total radiation and the wavelength distribution affected?

2. Explain how the Sun's surface temperature can be determined by measuring the flux of solar radiation outside Earth's atmosphere.

3. What is the solar constant and why does it vary over the year?

4. Which wavelength bands are electromagnetic radiation commonly divided into? In what way are these wavelength bands relevant in solar engineering?

5. How do the wavelength spectra for extraterrestrial radiation and radiation on Earth's surface differ? What is the explanation for these differences?

6. What is the air mass and how can it be approximated?

7. What is the AM1.5 spectrum? Why is it useful in solar engineering?

8. Explain the difference between diffuse and beam radiation. Why does solar radiation arrive at Earth's surface in these two forms? 
Chapter 5. Exercises

9. What quantities does the Angström equation relate?

10. What are the summer and winter solstices and the equinoxes? How are they related to the Sun's declination?

11. Describe, for latitudes between the tropics and the polar circles, how the Sun's apparent path across the sky changes between the winter and summer solstice.

12. Suggest a setup of measurement devices to monitor beam and diffuse radiation on the horizontal plane.

13. In what ways does solar time differ from local clock time?

14. What is the difference between the angle of incidence and the zenith angle of incidence? Why are these useful when determining the beam radiation incident on a surface?

15. Explain in a qualitative way what the view factor of a surface is.

16. Describe, qualitatively, how diffuse radiation is handled in the Hay and Davies model when:

(a) The weather is clear.

(b) The sky is completely overcast.

\section{Problems}

1. Compare two black-bodies, one with temperature $500 \mathrm{~K}$ and one with temperature $3000 \mathrm{~K}$.

(a) How do they differ in terms of peak wavelength?

(b) How much total radiation is emitted from each of them?

2. A futuristic concept known as a Dyson sphere envisions the entire Sun being covered with PV panels. If such a megastructure of panels was placed around the Sun, covering it entirely, what would be it's total power output, given a PV panel efficiency of $15 \%$ ? 
3. What would the solar constant at the planet Mars be if you know that it is $1367 \mathrm{~W} / \mathrm{m}^{2}$ at Earth? The mean distance between the Sun and Mars is 227.9 million kilometers.

4. Which zenith angle of incidence and Sun altitude angle correspond to AM1.5?

5. What is the difference in solar time (rounded to whole minutes) between the Swedish cities Göteborg $\left(57.70^{\circ} \mathrm{N}, 12.00^{\circ} \mathrm{E}\right)$ and Luleå $\left(65.55^{\circ} \mathrm{N}, 22.13^{\circ} \mathrm{E}\right)$ ?

6. Find a simplified formula for the angle of incidence of beam radiation on a plane that has azimuth angle $0^{\circ}$ and is tilted $90^{\circ}$. The expression should depend only on the declination, latitude and hour angle.

7. Three neighbours in Grästorp, Sweden $\left(58.33^{\circ} \mathrm{N}, 12.67^{\circ} \mathrm{E}\right)$, have installed PV arrays on their houses. These arrays are mounted directly on:

(a) A flat horizontal roof.

(b) A vertical wall facing south.

(c) A roof facing west, tilted $30^{\circ}$.

On the 23rd of July at 15:30 (standard clock time with summer hour shift), the neighbours are arguing about which array faces the Sun most directly at this time (i.e. for which the angle of incidence of beam radiation is smallest). Which one does? (A bit of help: 23rd of July is the 204th day of the year and the equation of time on this day is -6.47 minutes.)

8. For many practical applications involving solar energy, it is often useful to know when the Sun rises and sets.

(a) Derive expressions for the sunset and sunrise hour angle of the horizontal plane, i.e. the hour angle for which the Sun is precisely at the edge of a horizontally oriented surface. (Hint: The expressions will be symmetrical, differing only by the sign.)

(b) Use these expressions to find the standard clock time at which the Sun rises and sets in Grästorp on the same date as in the previous problem. 
9. If you know the zenith angle at solar noon, how should you orient a surface to face the Sun directly at this particular time?

10. Consider again the two tilted arrays in Problem 6.

(a) Which are the corresponding geometric factors for beam radiation at 15:30 on July 23rd?

(b) Assume that the area of each array is $10 \mathrm{~m}^{2}$. How much beam radiation would these arrays receive if the beam radiation on the horizontal plane is $500 \mathrm{~W} / \mathrm{m}^{2}$ at this time?

11. Find a simplified expression for the zenith incidence angle at solar noon on the day of the spring or autumn equinox.

12. On a spring day (which happens to be the day of the spring equinox) a group of engineering students have gathered for a picnic at solar noon in Eslöv, Sweden $\left(55.83^{\circ} \mathrm{N}, 13.30^{\circ} \mathrm{E}\right)$. One of them has brought a homebuilt electric stove powered by a PV array, but the others are unsure if it will be able to collect enough solar energy. At solar noon on this day, the horizontal plane receives $621 \mathrm{~W} / \mathrm{m}^{2}$ of global radiation, of which $236 \mathrm{~W} / \mathrm{m}^{2}$ are diffuse.

(a) It is decided that the PV array is oriented to collect as much beam radiation as possible at solar noon. Which tilt and azimuth angles should be chosen?

(b) Assuming that the ground has an albedo of $20 \%$, how much diffuse, beam and ground-reflected radiation does the array collect at this orientation? Use the Hay and Davies model for diffuse radiation. Extraterrestrial radiation with normal incidence on this day is $1376 \mathrm{~W} / \mathrm{m}^{2}$. 


\section{Answers to problems}

1. (a) 5.8 and $0.97 \mu \mathrm{m}$, respectively. (b) $3.5 \mathrm{~kW} / \mathrm{m}^{2}$ and $4.6 \mathrm{MW} / \mathrm{m}^{2}$.

2. $15 \%$ of the total radiative power of the Sun, or $5.79 \times 10^{25} \mathrm{~W}$. Notice also that this does not depend on the radius of the Dyson sphere.

3. $588.2 \mathrm{~W} / \mathrm{m}^{2}$.

4. Zenith angle $48.2^{\circ}$, Sun altitude angle $41.8^{\circ}$.

5. 41 minutes.

6. $\cos \theta_{z}=-\sin \delta \cos \phi+\cos \delta \sin \phi \cos \omega$.

7. Beam radiation on these three arrays has incidence angles (a) $45.4^{\circ}$, (b) $60.8^{\circ}$, (c) $58.8^{\circ}$. Array (a) wins.

8. (a) Sunset hour angle: $\omega=\arccos (-\tan \phi \tan \delta)$, sunrise hour angle: $\omega=-\arccos (-\tan \phi \tan \delta)$. (b) The Sun rises at 03:51 and sets at 20:40 (04:51 and 21:40 with summer hour shift). Note that this result may differ by several minutes from more detailed calculations of sunrise and sunset that take into account the angular diameter of the Sun and use other, more precise expressions for declination and the equation of time.

9. $\gamma=0, \beta=\theta_{z}$.

10. (a) 0.695 and 0.738 , respectively. (b) 3.48 and $3.69 \mathrm{~kW}$ per array.

11. $\theta_{z}=\phi$.

12. (a) $\gamma=0, \beta=55.83^{\circ}$. (b) $G_{b T}=685 \mathrm{~W} / \mathrm{m}^{2}, G_{d T}=302 \mathrm{~W} / \mathrm{m}^{2}$, $G_{g T}=27.2 \mathrm{~W} / \mathrm{m}^{2}$. 


\section{Appendix A}

\section{Derivation of the incidence angles of beam radiation}

\section{A.1 Zenith angle of incidence}

The zenith angle $\theta_{z}$ is the angle between the normal to the horizontal plane at a given location, and the incident beam radiation. The geometric components involved in finding an expression for $\theta_{z}$ are outlined in Figure A.1. The figure shows the local horizontal plane at some arbitrary location on Earth. The plane is defined by its inclination with respect to the celestial equator, which is the projection of Earth's equator on the celestial sphere. The angle between the equator plane and the normal vector of the local plane $(\boldsymbol{v})$ is equal to the latitude $\phi$ of the location.

Over the course of the day, Earth rotates with respect to the celestial sphere, making a complete cycle in one day. This movement is indicated by the hour angle $\omega$, which is the angle between the position of the current local meridian $L_{l o c}$ of Earth (as projected on the equator plane) and the meridian's position at solar noon, $L_{\text {noon }}$. The Sun's position on the celestial sphere is further defined by the declination $\delta$, which is the angular displacement of the Sun from the equator plane. The incidence angle $\theta_{z}$ can now be found as the angle between the vector $\boldsymbol{u}$, which points from the center of the celestial sphere to the Sun's position, and the normal vector $\boldsymbol{v}$ of the horizontal plane.

A convenient choice of coordinate system is to have the $x$ axis pointing in 


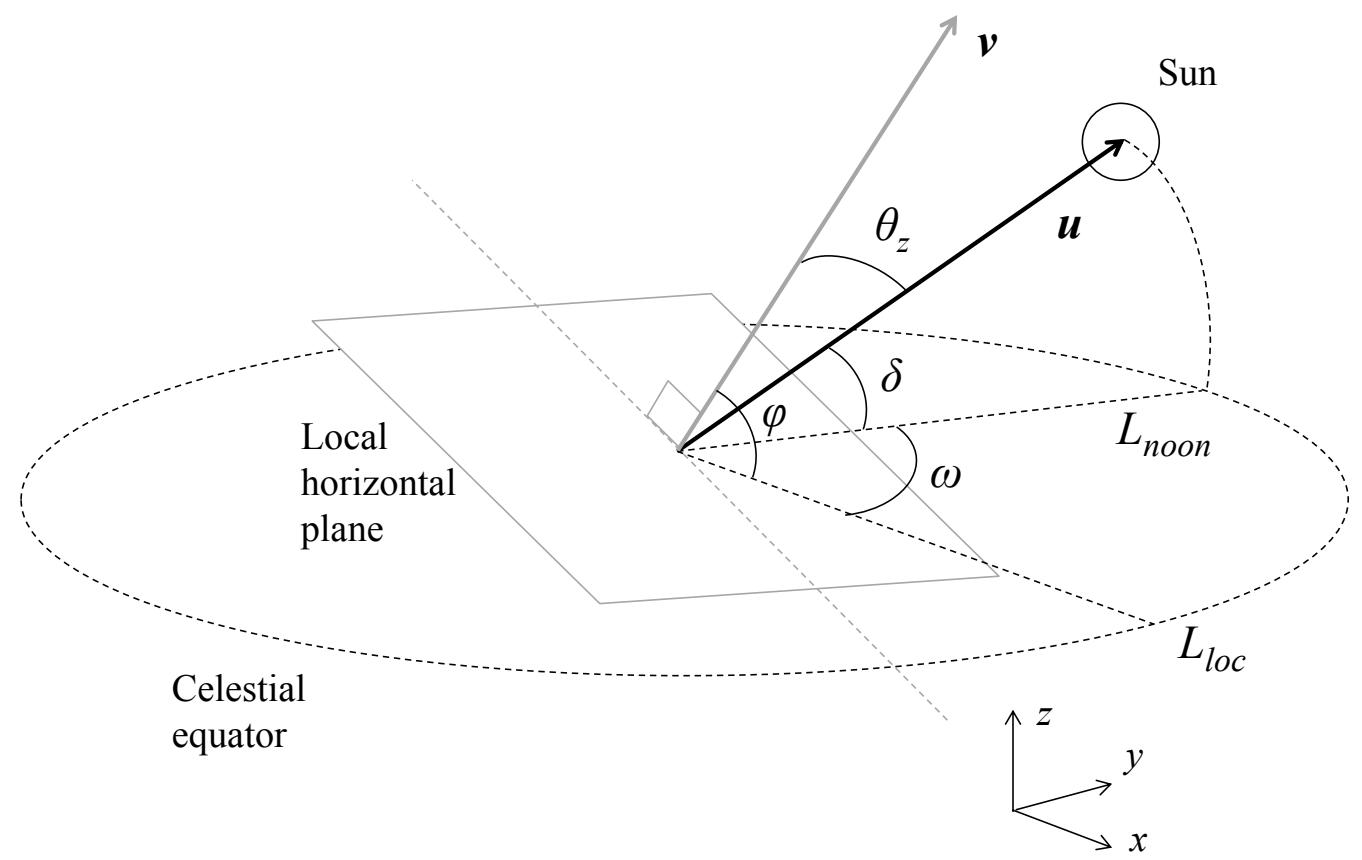

Figure A.1: Outline of the geometric relations between the Sun, the celestial equator and the horizontal plane of Earth at a given location.

the direction of $L_{l o c}$, the $y$ axis orthogonally to $x$ in the equator plane, and $z$ normal to the equator plane. This makes it possible to directly identify the components of the vectors $\boldsymbol{u}$ and $\boldsymbol{v}$ from the angles in Figure A.1. Since $\boldsymbol{v}$ by definition is in the $x z$ plane, its components are given solely by the angle $\phi$ as:

$$
\boldsymbol{v}=(\cos \phi, 0, \sin \phi)^{T}
$$

The vector $\boldsymbol{u}$ is displaced from the $x z$ plane by the angle $\omega$, and by the angle $\delta$ from the $x y$ plane, giving the following vector components:

$$
\boldsymbol{u}=(\cos \omega, \sin \omega, \tan \delta)^{T},
$$

which can be scaled into a unit vector by multiplying with $\cos \delta$ :

$$
\boldsymbol{u}=(\cos \delta \cos \omega, \cos \delta \sin \omega, \sin \delta)^{T},
$$

Now, since both $\boldsymbol{u}$ and $\boldsymbol{v}$ are unit vectors, the cosine of the angle $\theta_{z}$ between them are given by the dot product:

$$
\cos \theta_{z}=\boldsymbol{u} \bullet \boldsymbol{v}=\cos \phi \cos \delta \cos \omega+\sin \phi \sin \delta
$$




\section{A.2 Incidence angle on an arbitrarily oriented plane}

The incidence angle of beam radiation on an arbitrarily tilted plane is a bit more tricky to derive. Figure A.2 shows how a tilted surface is placed on the horizontal plane of Earth. The orientation of the tilted plane is defined by the azimuth angle $\gamma$ and the tilt angle $\beta$, defined with respect to the local meridian and the normal of the horizontal plane, respectively. What we now want to find is the angle $\theta$ between the Sun vector $\boldsymbol{u}$ and the normal vector $\boldsymbol{w}$ of the tilted plane.

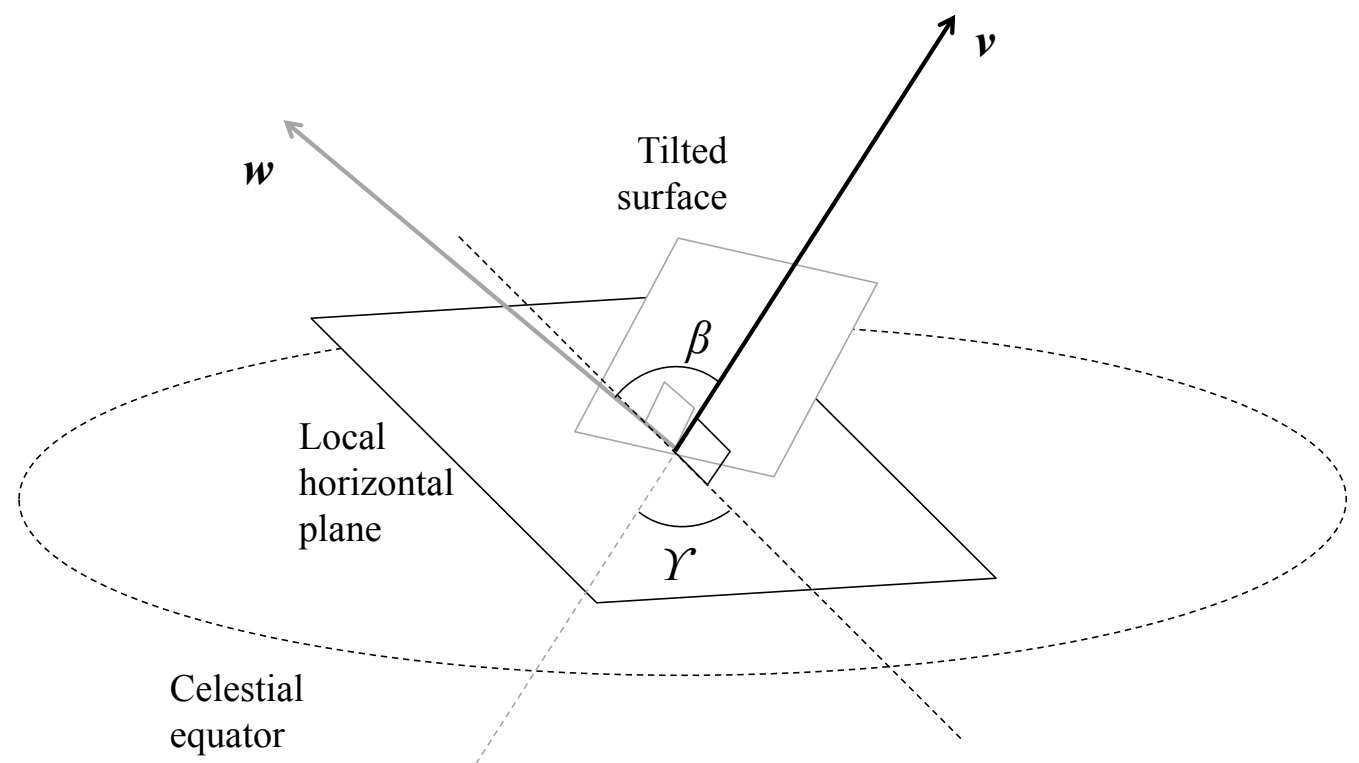

Figure A.2: The geometric relations between the horizontal plane and an arbitrarily oriented surface.

As Figure A.2 indicates, $\boldsymbol{w}$ can be found by first rotating $\boldsymbol{v}$ around the $y$ axis by the angle $\beta$, then rotating this vector around the original direction of $\boldsymbol{v}$ by the angle $\gamma$. A first step, to make this rotation conveniently, is to change the basis of the coordinate system so that $x$ is pointing in the direction of $\boldsymbol{v}$ while keeping the same $y$ direction. This means that the new base vectors are:

$$
\begin{aligned}
& \boldsymbol{i}=\boldsymbol{v}=(\cos \phi, 0, \sin \phi)^{T} \\
& \boldsymbol{j}=(0,1,0)^{T} \\
& \boldsymbol{k}=\boldsymbol{v} \times \boldsymbol{j}=(-\sin \phi, 0, \cos \phi)^{T}
\end{aligned}
$$


48 Appendix A. Derivation of the incidence angles of beam radiation

The transformation matrix for this operation is:

$$
A=\left(\begin{array}{lll}
\boldsymbol{i} & \boldsymbol{j} & \boldsymbol{k}
\end{array}\right)^{-1}=\left(\begin{array}{ccc}
\cos \phi & 0 & \sin \phi \\
0 & 1 & 0 \\
-\sin \phi & 0 & \cos \phi
\end{array}\right)
$$

i.e. a rotation around the $y$ axis by the latitude angle. Applying this transformation to the Sun vector $\boldsymbol{u}$ we get:

$$
\boldsymbol{u}^{\prime}=A \boldsymbol{u}=\left(\begin{array}{c}
\cos \phi \cos \delta \cos \omega+\sin \phi \sin \delta \\
\cos \delta \sin \omega \\
-\sin \phi \cos \delta \cos \omega+\cos \phi \sin \delta
\end{array}\right)
$$

Since the normal vector of the horizontal plane is equal to the new $i$ base vector, we simply have $\boldsymbol{v}^{\prime}=(1,0,0)$.

Now, to find the normal to the tilted plane, $\boldsymbol{w}^{\prime}$, we rotate $\boldsymbol{v}^{\prime}$ an angle $\beta$ around the $y$ axis, then an angle $\gamma$ around the new $x$ axis:

$$
\boldsymbol{w}^{\prime}=\left(\begin{array}{ccc}
1 & 0 & 0 \\
0 & \cos \gamma & -\sin \gamma \\
0 & \sin \gamma & \cos \gamma
\end{array}\right)\left(\begin{array}{ccc}
\cos \beta & 0 & \sin \beta \\
0 & 1 & 0 \\
-\sin \beta & 0 & \cos \beta
\end{array}\right) \boldsymbol{v}^{\prime}=\left(\begin{array}{c}
\cos \beta \\
\sin \gamma \sin \beta \\
-\cos \gamma \sin \beta
\end{array}\right)
$$

As before, we can now find the cosine of the angle $\theta$ as the dot product between $\boldsymbol{u}^{\prime}$ and $\boldsymbol{w}^{\prime}$ :

$$
\begin{aligned}
\cos \theta=\boldsymbol{u}^{\prime} \bullet \boldsymbol{w}^{\prime}= & \sin \delta \sin \phi \cos \beta \\
& -\sin \delta \cos \phi \sin \beta \cos \gamma \\
& +\cos \delta \cos \phi \cos \beta \cos \omega \\
& +\cos \delta \sin \phi \sin \beta \cos \gamma \cos \omega \\
& +\cos \delta \sin \beta \sin \gamma \sin \omega
\end{aligned}
$$




\section{Bibliography}

[1] R. Cohen (2010), Chasing The Sun: The Epic Story of the Star That Gives Us Life, London, GB: Simon \& Schuster.

[2] J.A. Duffie, W.A. Beckman (2006), Solar Engineering of Thermal Processes, Hoboken, NJ: Wiley.

[3] Solar Spectra, NREL: http://rredc.nrel.gov/solar/spectra/ (2019-0414).

[4] M. Iqbal (1983), Introduction to Solar Radiation, Toronto: Academic Press.

[5] M. Sengupta, A. Andreas (2010), Oahu Solar Measurement Grid (1-Year Archive): 1-Second Solar Irradiance; Oahu, Hawaii (Data), NREL Report No. DA-5500-56506. http://dx.doi.org/10.5439/1052451

[6] T. Persson (2000), Measurements of Solar Radiation in Sweden 19831998, RMK No. 89, Swedish Meteorological and Hydrological Institute (SMHI), Sweden.

[7] A. Ångström (1928), Recording solar radiation, messages from the Swedish meteorological and hydrological institute, 4:3.

[8] B. Sorensen (2004), Renewable Energy: Its Physics, Engineering, Use, Environmental Impacts, Economy and Planning Aspects, Amsterdam: Elsevier Academic Press.

[9] O. Beckman (1997), Angström: Father and Son, Acta Universitatis Upsaliensis C. Organisation och Historia 60, Uppsala University. 
[10] M. Paulescu, E. Paulescu, P. Gravila, V. Badescu (2013), Chapter 2, Weather Modeling and Forecasting of PV Systems Operation, Springer.

[11] J.T. Kiehl, K.E. Trenberth (1997), Earth's Annual Global Mean Energy Budget, Bulletin of the American Meteorological Society 78: 197-208.

[12] G.B. Smith, C.G. Granqvist (2011), Green Nanotechnology: Solutions for Sustainability and Energy in the Built Environment, Boca Raton, FL: CRC Press.

[13] D.T. Reindl, W.A. Beckman, J.A. Duffie (1990), Evaluation of hourly tilted surface radiation models, Solar Energy 45: 9-17.

[14] B. Perers (1999), The Solar Resource in Cold Climates, in: M. Ross, J. Royer (Eds.), Photovoltaics in Cold Climates, London: James \& James. 



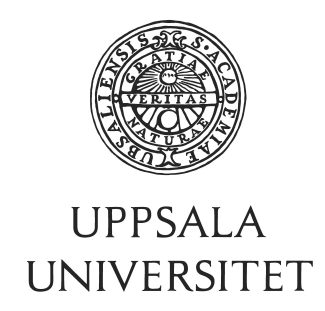

\section{Author biographies}

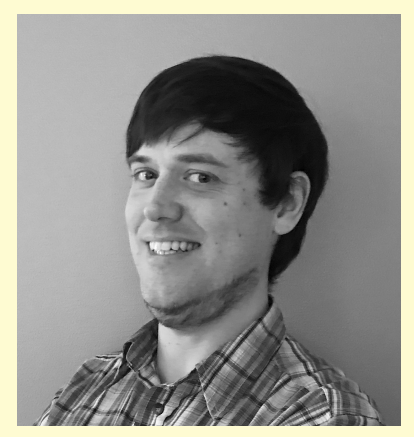

Joakim Widén is a Full Professor at the Department of Engineering Sciences at Uppsala University, Sweden, and Chair of the Division of Civil Engineering. His main area of expertise is spatiotemporal modeling of solar irradiance and its application in energy system operation and urbanscale building modeling.

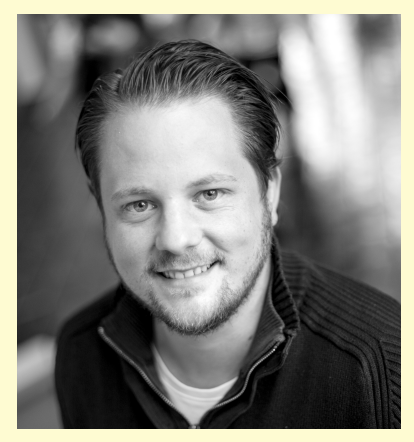

Joakim Munkhammar is an Associate Professor at the Department of Engineering Sciences at Uppsala University. His research is mainly focused on solar irradiance variability modeling and forecasting, as well as applications in energy systems such as electric vehicle charging. 